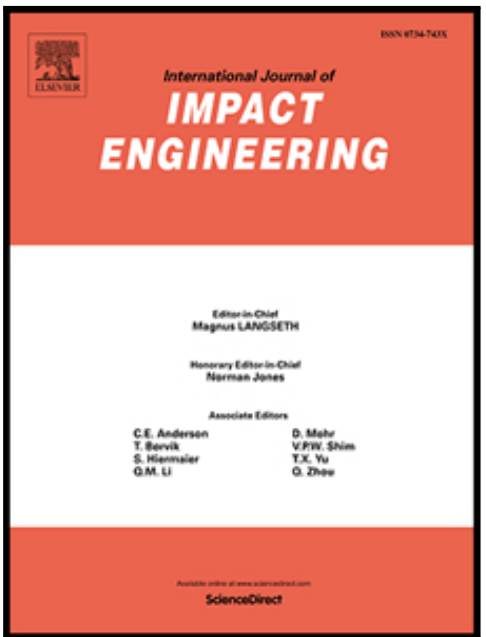

The influence of deformation limits on fluid-structure interactions in underwater blasts

Y. Yuan, P.J. Tan, K.A. Shojaei, P. Wrobel

PII:

S0734-743X(16)30078-1

DOI: 10.1016/j.jimpeng.2016.11.007

Reference: IE 2773

To appear in:

International Journal of Impact Engineering

Received date:

9 March 2016

Revised date:

13 October 2016

Accepted date:

14 November 2016

Please cite this article as: Y. Yuan, P.J. Tan, K.A. Shojaei, P. Wrobel, The influence of deformation limits on fluid-structure interactions in underwater blasts, International Journal of Impact Engineering (2016), doi: 10.1016/j.ijimpeng.2016.11.007

This is a PDF file of an unedited manuscript that has been accepted for publication. As a service to our customers we are providing this early version of the manuscript. The manuscript will undergo copyediting, typesetting, and review of the resulting proof before it is published in its final form. Please note that during the production process errors may be discovered which could affect the content, and all legal disclaimers that apply to the journal pertain. 


\section{Highlights}

2 - The classical FSI problem is revisited for a fully clamped elastoplastic beam

3 - Beneficial effects of FSI depends on the transverse deflection and time to detachment

- Impulse transmitted is reduced by increasing aspect ratio in mode I deformation

- Decreasing aspect ratio always reduces impulse transmitted in modes II and III

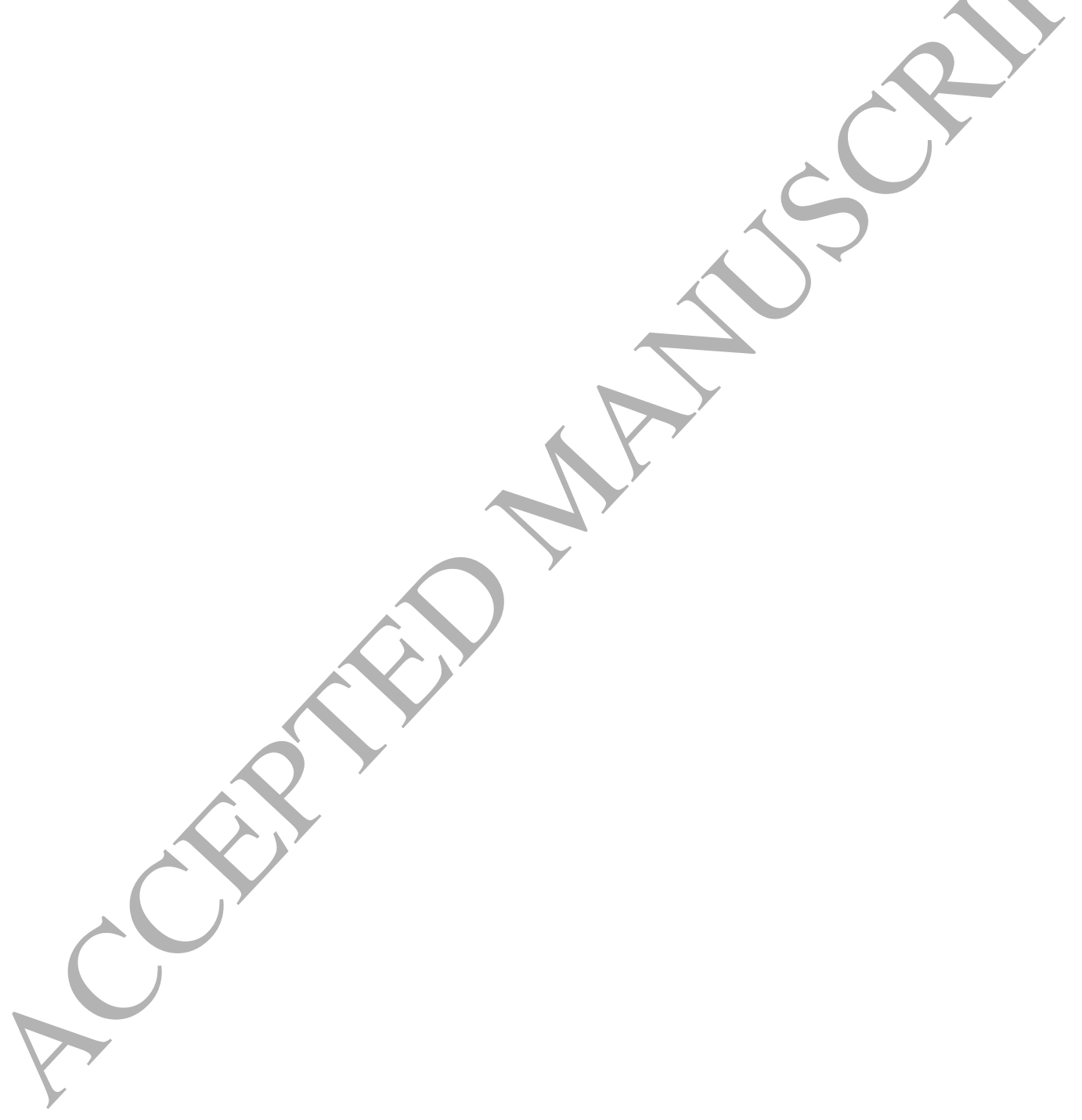




\title{
The influence of deformation limits on fluid-structure interactions in underwater blasts
}

\author{
Y. Yuan ${ }^{\dagger}$, P. J. Tan*, K. A. Shojaei, P. Wrobel \\ Department of Mechanical Engineering, University College London, Torrington Place, \\ London WC1E $7 J E, U K$
}

\begin{abstract}
\end{abstract}
This paper revisits a classical fluid-structure interaction (FSI) problem on the momentum and energy transfer to a structure from an underwater blast. Hitherto, the majority of analytical models assume a rigid (non-deformable) and frée-standing (unsupported) structure where resistance to its translational motion - apart from that offered by its inertial mass - comes from 'ad-hoc' backing spring(s) introduced to simulate compression of the fluid medium and/or the resistance to transverse deformation encountered by a real structure. These limitations/assumptions are relaxed in this paper by adopting a physically realistic fully-clamped ductile beam system that takes into account large elasto-plastic deformation, limits to material deformation, boundary compliance and boundary failure; the analytical framework was developed previously by Yuan et al. [1]. By coupling the fluid (water) domain to the analytical model of the ductile beam system, the momentum and energy transferred by the blast wave are critically re-evaluated for non-impulsive loading régime; in particular, on how the beam's deformation mode and boundary compliance affects fluid and structure interaction, up until the point of complete beam detachment from its supports. Detailed finite-element models were also developed to simulate the interactions between the fluid and structural beam where predictions were in good agreement with those by the analytical model. Sensitivity analyses were carried out that offer new insights on the influence of the beam's aspect ratio and inertial mass.

Keywords: Fluid-structure interaction, Underwater blast, Limits to deformation, Energy and momentum transfer, Ductile damage

${ }^{\dagger}$ Currently at Department of Automotive Engineering, Tsinghua University, Beijing, China

${ }^{*}$ Corresponding author Tel: +44 (0)20 76793754

Email address: pj.tan@ucl.ac.uk (P. J. Tan) 


\section{Introduction}

The beneficial effects of fluid-structure interaction (FSI) in reducing the impulse imparted to a rigid free-standing plate from an underwater blast are well-known. Taylor have shown that the momentum acquired by the plate reduces dramatically with its inertial mass: a direct consequence of early cavitation at the fluid-structure interface [2]. Over the past two decades, this 'peculiar' property has been extensively exploited to design sandwich panels with a greater resistance, compared to its monolithic equivalent of the same mass, to underwater blast loadings [3-9]. However, the majority of these studies on FSI, including those on sandwich panels, have largely ignored limits to deformation - frøm its supports and the structural material - which could potentially limit the external validity of any model predictions. There are two important factors that influence energy and momentum transfer to a submerged structure in underwater blasts: (1) development and evolution of cavitation zone(s); and, (2) limits to material deformation, boundary (supports) compliance and its failure. In the present study, we shall be concerned only with FSI in the "pre-boundary failure' régime, i.e. before the complete detachment of the structure from its supports.

\begin{tabular}{|c|c|c|c|}
\hline \multicolumn{4}{|c|}{ Nomenclature } \\
\hline$A$ & Cross-section area of beam & & Fully plastic membrane force \\
\hline$B$ & Width of beam & $\bar{N}$ & $N / N_{0}$ \\
\hline$c_{w}$ & Acoustic wave speed in water & $p_{I}$ & Incident pressure wave \\
\hline$D$ & Damage variable & $p_{s}$ & peak incident pressure \\
\hline $\bar{E}^{F}$ & $\begin{array}{l}\text { Non-dimensional maximum to- } \\
\text { tal energy transmitted to free- } \\
\text { standing beam }\end{array}$ & $p_{R 1}$ & Reflected pressure wave \\
\hline$E^{T}, E^{K}$ & $\begin{array}{l}\text { Transmitted energy and ki- } \\
\text { netic energy of the elasto plas- } \\
\text { tic beam }\end{array}$ & $p_{R 2}$ & Rarefaction pressure wave \\
\hline $\bar{E}^{T}, \bar{E}^{K}$ & Non-dimensional maximum & $p_{\text {Int }}$ & Interface pressure \\
\hline & $\begin{array}{l}\text { Bending, shear, membrane en- } \\
\text { ergy obtained from rotational, }\end{array}$ & $\tilde{p}_{\text {Int }}$ & Average interface pressure \\
\hline$E_{B}^{b}, 1$ & $\begin{array}{l}\text { axial and vertical springs } \\
\text { Bending, shear, membrane en- } \\
\text { ergy of the elastoplastic beam }\end{array}$ & $Q$ & Transverse shear force \\
\hline & $\begin{array}{l}\text { Incident energy of blast wave } \\
\text { per unit area }\end{array}$ & $Q_{0}$ & Fully plastic shear force \\
\hline$H$ & Beam thickness & $t_{1}, t_{2}, t_{3}$ & $\begin{array}{l}\text { Termination time of Phases I, } \\
\text { II and III }\end{array}$ \\
\hline & Incident impulse per unit area & $t_{c}$ & Cavitation time \\
\hline$I^{T}, I^{K}$ & $\begin{array}{l}\text { Transmitted impulse, momen- } \\
\text { tum }\end{array}$ & $t_{i}$ & Decay constant \\
\hline
\end{tabular}




\begin{tabular}{|c|c|c|c|}
\hline $\bar{I}^{F}$ & $\begin{array}{l}\text { Maximum total impulse per } \\
\text { unit area of free-standing beam }\end{array}$ & $\hat{W}$ & Average transverse deflection \\
\hline $\bar{I}^{T}, \bar{I}^{K}$ & Non-dimensional maximum $I^{T}$ & $W_{0}$ & Maximum mid-span deflection \\
\hline $\bar{I}_{1}^{T}$ & $\begin{array}{l}\text { Maximum non-dimensional } \\
\text { transmitted impulse }\end{array}$ & $W_{B}, W_{S}$ & Deflection at mid-span \& support \\
\hline $\bar{I}_{2}^{T}$ & $\begin{array}{l}\text { Reduction of transmitted im- } \\
\text { pulse due to failure }\end{array}$ & $Z$ & Lagrangian coordinates \\
\hline $\bar{I}_{3}^{T}$, & $\begin{array}{l}\text { Reduction of transmitted im- } \\
\text { pulse due to deformation }\end{array}$ & $\beta$ & $\begin{array}{l}\text { Ratio of the plastic work ab- } \\
\text { sorbed through shear deforma- } \\
\text { tion to the total plastic work } \\
\text { done }\end{array}$ \\
\hline$I^{*}$ & Non-dimensional impulse & $\beta_{c}$ & $\begin{array}{l}\text { Critical value of } \beta \text { separating } \\
\text { modes II and III }\end{array}$ \\
\hline$\hat{I}$ & Impulse per unit area & $\beta_{w}$ & FSI index) \\
\hline$K_{\phi}$ & Rotational spring stiffness & $\Delta W_{0}$ & Relative mid-span displacement \\
\hline$L$ & Half length of beam member & & State variable for ductile and \\
\hline$L_{e}$ & Characteristic length of the & & le mode functions \\
\hline$L_{w}$ & $\begin{array}{l}\text { first-order element in FE } \\
\text { Length of water column in FE }\end{array}$ & & Density of beam material \\
\hline$M_{0}$ & Fully plastic bending moment & & Density of water \\
\hline$N$ & Membrane force & $\sigma_{Y}$ & Static yield strength \\
\hline
\end{tabular}

Treating water as a linear-elastic medium, Kennard [10] found that if the pressure at any point drops below the cavitation limit, two 'breaking fronts' emerge from there and propagate in opposite directions, creating an expanding pool of cavitated liquid. These breaking fronts can arrest, invert their direction of motion and become 'closing fronts', forcing the contraction of the cayitation zone. Schiffer et al. [11] studied the effects of initial hydrostatic pressure on cavitation for a rigid plate with a linear backing spring. Their model is able to capture the propagation of both breaking and closing fronts, as well as their interactions with the structure, in a blast event. It was found that increasing hydrostatic pressure reduces the transmitted impulse since it moves the point of incipient cavitation away from the structure; however, reducing inertial mass does not always lead to a reduction in the transmitted impulse whilst increasing the supporting stiffness always will. Schiffer and Tagarielli [12] further reported a 'double-cavitation' event where early plate deformation, due to the propagation of flexural waves, gives rise to a localised cavitation zone at the fluid-structure interface and in the central portion of the plate. This zone quickly collapses upon coalescence of the flexural wave at the centre. Subsequent plate deformation induces an additional cavitation at a finite distance from the plate as previously described.

It is, as yet, unclear how limits to material deformation, support compliance and support failure affects previously known results since the impulse imparted by an underwater blast load- 

5 .

ing is often sufficiently intense to induce significant plastic deformation in a structure with which it interacts leading to, in extreme circumstances, a loss of structural integrity through partial/complete detachment from its support. In this paper, the limitations/assumptions of previous studies are relaxed by considering a fully-clamped ductile beam system - the analytical framework for this was developed previously by Yuan et al. [1] in a companion paper. The model of the ductile beam system is able to capture the three different modes of deformation observed in blast experiments, the initiation and evolution of damage with increasing transverse beam deflection, and its consequential detachment - by fracture - from the supports. By coupling the fluid (water) domain to the aforesaid model of the ductile beam system, the momentum and energy transferred by the blast wave are critically reevaluated for the coupled, non-impulsive loading regime; in particular, on how the beam's deformation mode and boundary compliance affects the fluid and structure interaction, or vice-versa, before the onset of boundary failure, defined as the complete detachment of the beam from its supports.

The outline of this paper is as follows: Section 2 summaries the key features of the ductile beam system developed in [1] and outlines the fluid-structure coupling strategy; details of the three-dimensional (3D) FE model are given in Section 3; Section 4 compares the predictions of the analytical and FE models; and, finally, results for the elasto-plastic and rigid free-standing beams are compared and sensitivity analyses carried out to elucidate the dependence of the model predictions on the beam's aspect ratio and inertial mass in Section

\section{Analytical model [1]}

The analytical framework for the fully-clamped ductile beam system - developed by Yuan et al. [1] in a separate study - are briefly outlined with particular attention paid to highlighting the key elements that had been introduced to incorporate elasto-plastic constitutive behaviour, boundary compliance and boundary failure. This is followed by details on coupling strategy between the fluid domain and beam system, and on the limitations of the current FSI model.

\subsection{Fully clamped ductile beam system - key features}

The ductile beam system incorporates the following: (1) large elasto-plastic deformation with catenary action; (2) interactions between bending, membrane stretch and transverse shear; and, (3) limits to deformation through a loss of integrity at the support and the subsequent beam detachment by rupture. Figure 1 shows a schematic of the slender beam supported at each end by three springs (one rotational and two axial). The pressure loading $p(x, t)$ is assumed to always impinge normally over the span of the beam regardless of its subsequent transverse deflection $[13,14]$. Hence, a plane of geometric and loading symmetry exists at the mid-span of the beam $(x=0)$, allowing one-half of the beam to be analysed. 
The beam - made of a rate-independent, elastic perfectly-plastic material - has a total length $2 L$ and a uniform rectangular cross-section of thickness $H$ and width $B$ where $L / H \gg 1$. The rotational spring has elasto-plastic characteristics to model the end rotation of the beam and the subsequent formation of a plastic hinge. Both the axial and vertical springs have rigid-plastic characteristics to model the 'plastic stretch' and 'plastic shear sliding' actions at the support, respectively.

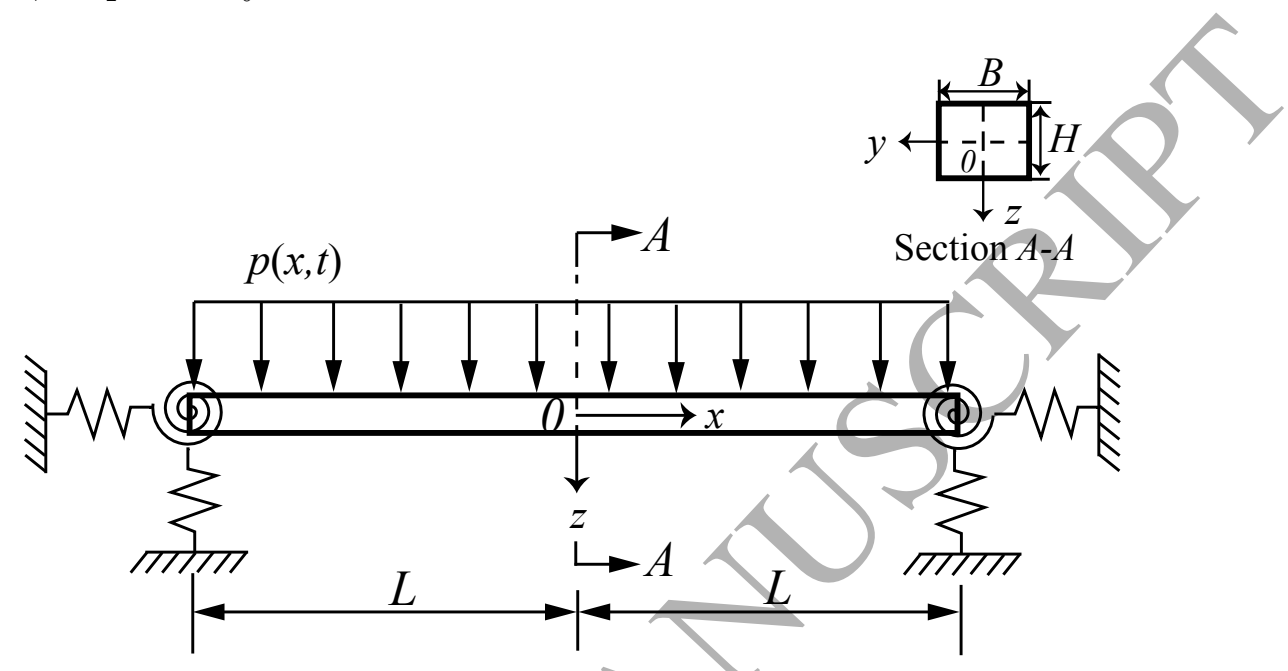

Figure 1: Schematic of the fully clamped ductile beam system by Yuan et al. [1]. A plane of symmetry exists along $x=0,-B / 2 \leq y \leq B / 2,-H / 2 \leq z \leq H / 2$ so that only the right-half needs to be analysed.

\subsubsection{Fully plastic stress condition, damage and failure criteria}

Plastic limit function, damage and failure criteria are first established as functions of nondimensional fully plastic generalised stresses, viz. $\bar{M}\left(\triangleq M / M_{0}\right), \bar{N}\left(\triangleq N / N_{0}\right)$ and $\bar{Q}(\triangleq$ $\left.Q / Q_{0}\right)$ where $M_{0}=\sigma_{Y} B H^{2} / 4, N_{0}=\sigma_{Y} B H$ and $Q_{0}=\sigma_{Y} B H / \sqrt{3}$ are the fully plastic bending moment, membrane force and transverse shear force, respectively; and, $\sigma_{Y}$ is the uniaxial static yield strength of the beam material. For a beam of rectangular cross-section, its fully plastic limit function $\psi^{p}$ can be written as [15]

$$
\psi^{p}=|\bar{M}| \sqrt{1-\bar{Q}^{2}}+\bar{N}^{2}+\bar{Q}^{2}-1 .
$$

Consequently, a plastic hinge forms at any cross section where the fully plastic stress condition $\psi^{p}=0$ is reached.

Damage shall refer to the onset and subsequent degradation of the generalised stresses in the beam or its supports, or both. Figure 2 gives a schematic of the variation of generalised stresses $(\bar{M}$ or $\bar{N}$ or $\bar{Q})$ versus effective strain $\epsilon_{\text {eff }}$ defined on any cross section of the beam, including at its supports. Point $c$ denotes the generalised stress state at a cross-section when the damage initiation criterion is satisfied as follows:

$$
\omega_{d}=\frac{\epsilon_{\mathrm{eff}}}{\epsilon_{d}}=1
$$


where $\omega_{d}$ is a state variable that increases monotonically with effective strain $\epsilon_{\text {eff }}$, and $\epsilon_{d}$ is the effective strain corresponding to the onset of damage. Beyond this, progressive softening of the non-dimensional generalised stresses occur in accordance to

$$
|\bar{M}|=\left|\bar{M}^{f}\right|(1-D), \quad \bar{N}=\bar{N}^{f}(1-D) \quad \text { and } \quad \bar{Q}=\bar{Q}^{f}(1-D)
$$

where $D$ is a damage variable; $\bar{M}^{f}, \bar{N}^{f}$ and $\bar{Q}^{f}$ are the non-dimensional bending moment, membrane force and transverse shear force at the onset of damage, respectively. For simplicity, a linear evolution of the damage variable $D$ with effective strain $\epsilon_{\text {eff }}$ is adopted as follows [16]:

$$
D=\frac{\epsilon_{\mathrm{eff}}-\epsilon_{d}}{\epsilon_{r}-\epsilon_{d}}
$$

where $\epsilon_{r}$ is the rupture strain to be obtained from a uniaxial tensile test. This definition ensures that all the generalised stresses reduce to zero when $D=1$.

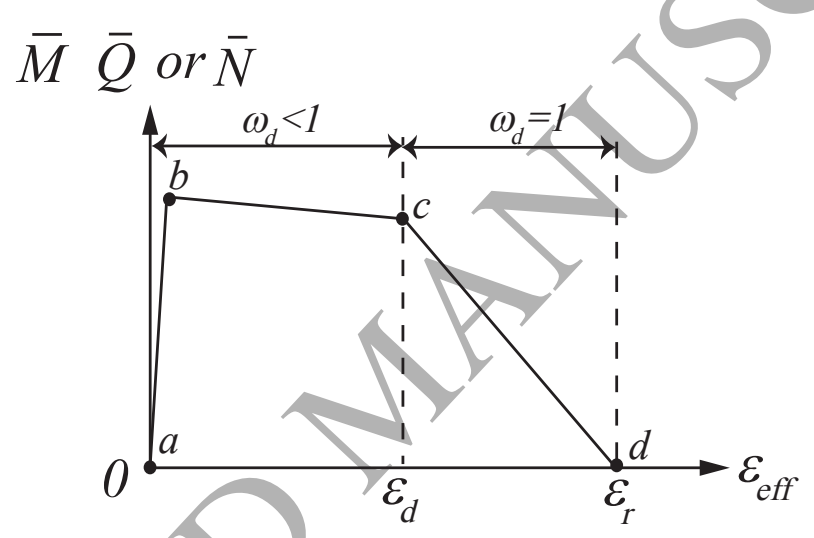

Figure 2: Schematic on how the non-dimensional generalised stresses vary with effective strain pre- $\left(\omega_{d}<1\right)$ and post- $\left(\omega_{d}=1\right)$ damage initiation.

Failure refers to a complete loss of load carrying capacity by the beam through detachment from its supports. Since the effective strain $\epsilon_{\text {eff }}$ is always greatest at the support $(x=L)$ see Section 2.1.2- a failure criterion needs only to be defined there where damage must first initiates and where failure would subsequently occur $[17,18]$. The criteria delineating the different modes of failure, corresponding to those previously identified by Menkes and Opat [19], are as follows:

$$
\begin{array}{rll}
\text { Mode I : } & D<1, & \omega_{s}<1 \\
\text { Mode II : } & D=1, & \omega_{s}<1 \\
\text { Mode III : } & D=1, & \omega_{s} \geq 1 .
\end{array}
$$

The state variable $\omega_{s}$ is expressed as

$$
\omega_{s}=\frac{\beta}{\beta_{c}}
$$


where $\beta$ is the ratio of the plastic work absorbed through shearing deformation to the total plastic work done by all the other stress components given by [20]

$$
\beta=\frac{E_{S}^{s}}{E_{S}^{s}+E_{S}^{b}+E_{S}^{m}}
$$

where $E_{S}^{s}$ is the shear strain energy (vertical axial spring); $E_{S}^{b}$ is the bending strain energy (rotational spring); $E_{S}^{m}$ is the membrane strain energy (horizontal axial spring), and, $\beta_{c}$ is the critical $\beta$ value delineating the transition from mode II to III to be given later.

\subsubsection{Effective strain $\epsilon_{\mathrm{eff}}$ and material properties}

The effective strain $\epsilon_{\text {eff }}$ in Eqs. 2 and 4 will now be approximated. In general, the effective strain $\epsilon_{\text {eff }}$ on any cross-section is given by $[17,18]$

$$
\epsilon_{\mathrm{eff}}=\sqrt{\frac{2}{9}\left[\left(\epsilon_{x x}-\epsilon_{y y}\right)^{2}+\left(\epsilon_{y y}-\epsilon_{z z}\right)^{2}+\left(\epsilon_{x x}-\epsilon_{z z}\right)^{2}+\frac{3}{2}\left(\gamma_{x z}^{2}+\gamma_{z y}^{2}+\gamma_{x y}^{2}\right)\right]} .
$$

For slender member where its thickness $H$ is much smaller compared to its half-length $L$, out-of-plane warping of cross-section is negligible; hence, plane sections remain plane [15]. Therefore, it is reasonable to write $\gamma_{x y}=\gamma_{y z}=0[17,18]$. If the material in a dynamic uniaxial test where $\epsilon_{x x}>0$ obeys the incompressibility relation $\epsilon_{x x}+\epsilon_{y y}+\epsilon_{z z}=0$, then $\epsilon_{y y}=\epsilon_{z z}=-\epsilon_{x x} / 2$ [21]. Thus, Eq. 8 simplifies to

$$
\epsilon_{\mathrm{eff}}=\sqrt{\epsilon_{x x}^{2}+\frac{1}{3} \gamma_{x z}^{2}}
$$

The maximum total in-plane strain $\epsilon_{x x}$ on any cross-section $x$ comprises of two parts given by $[17,21]$

$$
\epsilon_{x x}=\epsilon_{m}+\epsilon_{b}
$$

where the membrane strain $\epsilon_{m}$ and bending strain $\epsilon_{b}$ may be expressed, respectively, as functions of the transverse mid-span displacement of the beam $W_{B}$ as follows:

$$
\epsilon_{m}=2\left(\frac{W_{B}}{L}\right)^{2}\left(\frac{x}{L}\right)^{2}
$$

and

$$
\epsilon_{b}=\frac{W_{B} H}{L^{2}} .
$$

Just as in [21], a linear variation of the membrane strain $\epsilon_{m}$ along the half-length of the beam is also assumed here for simplicity.

Following $[17,18,22,23]$, the transverse shear strain $\gamma_{x z}$ is neglected in the beam member whist, at the support, it depends on the plastic shear sliding distance over the shear band. Thus, on any cross-section of the ductile beam, it follows that

$$
\gamma_{x z}=\left\{\begin{aligned}
0 & \text { if } 0 \leq x<L \\
W_{S} /(l / 2) & \text { if } x=L
\end{aligned}\right.
$$


where $W_{S}$ is the plastic shear sliding displacement and $l$ is the width of the shear band. Slip-line field analysis of a rigid-plastic beam with rectangular cross-section and thickness $H$ by Nonaka [24] showed that the width of the shear band ranges between $H \leq l \leq 2 H$ if the maximum transverse beam deflection is between 0 to $H$. The latter $(l=2 H)$ corresponds to the onset of membrane (or catenary) response in the beam. Since large beam deflection invariably leads to membrane effects, a value of $l=2 H$ is adopted here by following [23, 25]. Substituting Eqs. 10 - 13 into Eq. 9, gives an approximate expression for the effective strain on any cross-section $x$ of the beam system as follows:

$$
\epsilon_{\mathrm{eff}}=\left\{\begin{aligned}
2\left(\frac{W_{B}}{L}\right)^{2}\left(\frac{x}{L}\right)^{2}+\left(\frac{W_{B} H}{L^{2}}\right) & \text { if } 0 \leq x<L \\
\sqrt{\left[2\left(\frac{W_{B}}{L}\right)^{2}+\left(\frac{W_{B}}{L}\right)\left(\frac{H}{L}\right)\right]^{2}+\frac{1}{3}\left(\frac{W_{S}}{H}\right)^{2}} & \text { if } x=L
\end{aligned}\right.
$$

It is worth re-emphasising that the effective strain is always greatest at the supports $(x=L)$ since its two constituent components (total in-plane $\epsilon_{x x}$ and transverse shear strains $\gamma_{x z}$ ) are both highest there; this is also in agreement with results in $[17,18]$. Expressions for $W_{B}$ and $W_{S}$ are to be derived later in Section 2.1.3.

Table 2 lists the material properties for Aluminium 6061-T6 beams used by Menkes and Opat [19]; they may be assumed to be strain rate insensitive [26]. In this study, we adopt a rupture strain of $\epsilon_{r}=0.5$ [20] and an effective strain corresponding to the onset of damage at $\epsilon_{d}=0.38$ - both were calibrated to the experimental data of Menkes and Opat [19]. $\mathrm{Yu}$ and Chen [22] have previously shown that $\beta_{c}$ obtained for a square yield criterion is greater than that obtained for an interactive yield criterion $\left(\beta_{c}=0.45\right)$ by Shen and Jones [20]. Furthermore, they established that $\beta_{c}$ is a material-dependent parameter which is independent of geometry. Since the present study adopts an interactive fully plastic limit function and the same material properties as $[20,22]$ were used, it is reasonable to adopt $\beta_{c}=0.45$.

Table 2: Material properties for the Aluminium 6061-T6 beam [19]

\begin{tabular}{cccc}
\hline $\begin{array}{c}\text { Density, } \\
\rho\left(\mathrm{kg} / \mathrm{m}^{3}\right)\end{array}$ & $\begin{array}{c}\text { Young's modulus, } \\
E(\mathrm{GPa})\end{array}$ & $\begin{array}{c}\text { Static yield } \\
\text { stress, } \sigma_{Y}(\mathrm{MPa})\end{array}$ & $\begin{array}{c}\text { Poisson's } \\
\text { ratio }\end{array}$ \\
\hline 2686 & 69 & 283 & $1 / 3$
\end{tabular}

\subsubsection{Equations of motion}

Following $[13,14,27]$, the deformation of the beam is divided into three phases - see Fig. 3 - according to the sequence of plastic hinge formation as follows: (1) Phase $1\left(0<t \leq t_{1}\right)$ when plastic hinge does not form (or the fully plastic stress condition is not met) anywhere in the beam, i.e. $\psi^{p}<0$; (2) Phase II $\left(t_{1}<t \leq t_{2}\right)$ - when a stationary plastic hinge forms at the support; (3) Phase III $\left(t_{2}<t \leq t_{3}\right)$ - when a travelling plastic hinge $A$ moves towards, 


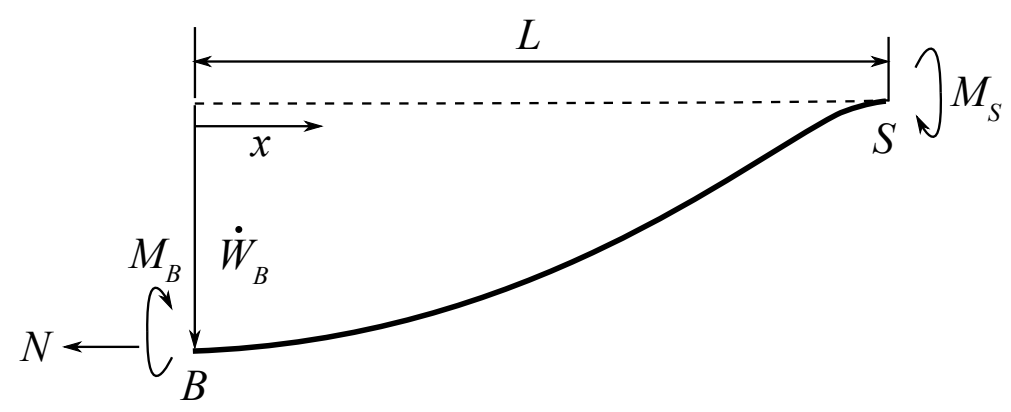

(a) $0<t \leq t_{1}$

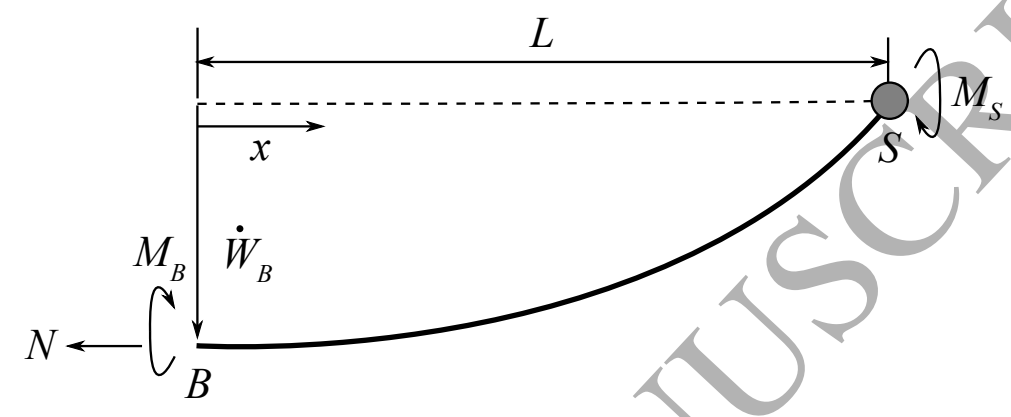

(b) $t_{1}<t \leq t_{2}$

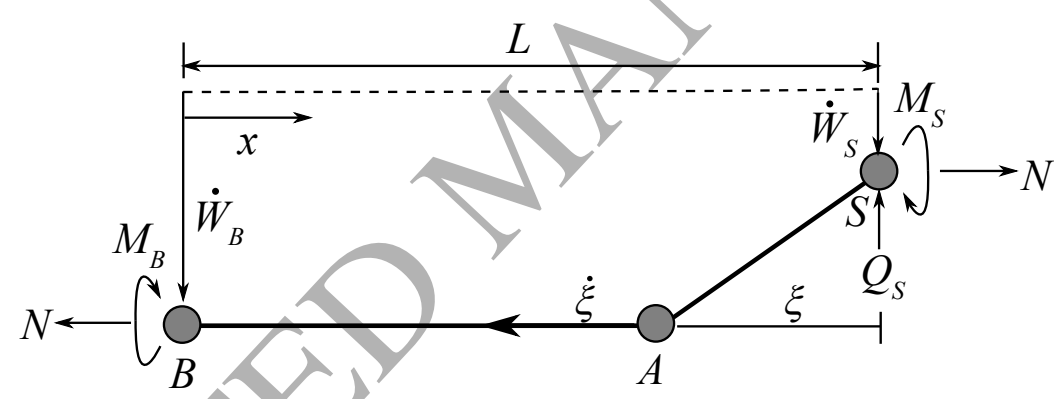

(c) $t_{2}<t \leq t_{3}$

Figure 3: Schematic of the transverse displacement profile for the right-half of the ductile beam system in [1] : (a) Phase I, (b) Phase II and (c) Phase III.

and coalesce with, the existing stationary hinge at the mid-span of the beam, ending up in a final two-hinge collapse configuration.

In each phase, the transverse displacement of the beam at a point $x(x>0)$ along the beam member and at its support is approximated as a sum of $n$ generalised displacements and mode functions given by [28]

$$
W(x, t)=\sum_{i=1}^{n} \phi_{i}(x) w_{i}(t)
$$

where the partial functions $\phi_{i}(x)$ are admissible mode functions that satisfy the geometric boundary conditions and the temporal functions $w_{i}(t)$ are generalised transverse displace- 
191

ments. The displacement at the mid-span and supports are, respectively,

$$
W_{B}(t)=W(x=0, t) \quad \text { and } \quad W_{S}(t)=W(x=L, t) .
$$

where $M_{i j}$ is the generalised mass given by

$$
M_{i j}=\left\{\begin{array}{rr}
m \int_{0}^{L} \phi_{i}(x) \phi_{j}(x) \mathrm{d} x & \text { if } i \neq j \\
m \int_{0}^{L} \phi_{i}^{2}(x) \mathrm{d} x & \text { if } i=j
\end{array},\right.
$$

The corresponding velocity $(\dot{W})$ and acceleration $(\ddot{W})$ fields also uses the same partial functions given by

$$
\dot{W}(x, t)=\sum_{i=1}^{n} \phi_{i}(x) \dot{w}_{i}(t) \quad \text { and } \quad \ddot{W}(x, t)=\sum_{i=1}^{n} \phi_{i}(x) \ddot{w}_{i}(t) .
$$

The differential equations governing $w_{i}$ can be obtained by substituting the Lagrangian ( $\triangleq E_{k}+V$ where $E_{k}$ and $V$ is total kinetic and potential energy of the system, respectively) for the structural beam system into the well-known Lagrange equation of the 2 nd kind, and simplifying, to give [1]

$$
\sum_{j=1}^{n} M_{i j} \ddot{w}_{j}+\frac{\partial V}{\partial w_{i}}=\int_{0}^{L} p(x, t) \phi_{i}(x) \mathrm{d} x,\langle i=1,2, \ldots, n
$$

$m$ is the mass per unit length; $V\left(\triangleq E_{S}^{b}+E_{S}^{s}+E_{S}^{m}+E_{B}^{b}+E_{B}^{m}\right)$ is the total potential energy of the beam system; $E_{B}^{b}$ and $E_{B}^{m}$ are the bending and membrane strain energies of the beam member, respectively; $E_{S}^{s}, E_{S}^{b}$ and $E_{S}^{m}$ are the shear, bending and membrane strain energies associated with the vertical, rotational and axial springs, respectively, at the supports. Note that subscripts $S$ and $B$ denote support and beam member, respectively; whilst, superscripts $s, b$ and $m$ denote shear, bending and membrane, respectively. The key to obtaining the governing equations of motion in Eq. 18 is to derive the various strain energy components $\left(E_{S}^{b}, E_{S}^{s}, E_{S}^{m}, E_{B}^{b}\right.$ and $\left.E_{B}^{m}\right)$ for each Phase - they are summarised in Appendix A.

\subsection{Coupling of fluid domain to the structure}

Consider a rightward-propagating planar pressure pulse that travels at a constant speed $c_{w}(=1498 \mathrm{~m} / \mathrm{s})$ in a fluid of density $\rho_{w}\left(=1000 \mathrm{~kg} / \mathrm{m}^{3}\right)$, and impinging normally on the structural beam system shown schematically in Fig 4. It is convenient to define a spatial coordinate $Z$ in the un-deformed configuration with $Z=0$ corresponding to the location of the fluid and structure interface, i.e. $Z=z+H / 2$. Assuming an exponentially decaying blast pulse with a peak pressure $p_{s}$ and time constant $t_{i}$, then the incident pressure wave at any arbitrary point (of coordinate $Z$ and $x$ ) at the time $t$ may be expressed as [2]

$$
p_{I}(Z, t)=p_{s} e^{-\left(t-Z / c_{w}\right) / t_{i}} .
$$




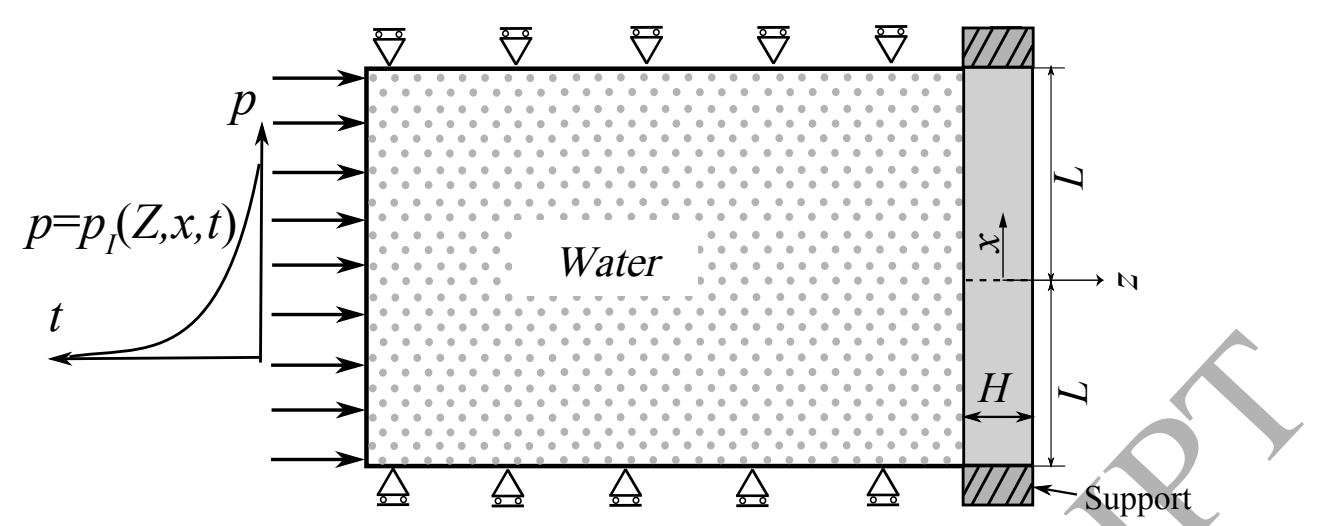

Figure 4: Schematic of an exponentially decaying wave propagating to the right towards a clamped elastoplastic beam system.

The incident pulse duration is considered to be infinitely long, i.e. $t_{d}=\infty$. If the target structure is rigid and fixed in space, then the reflected wave would be

$$
p_{R 1}(Z, t)=p_{s} e^{-\left(t+Z / c_{w}\right) / t_{i}}
$$

corresponding to a perfect reflection of the incident wave in the negative $Z$ direction. Since the beam is not rigid, the impingement of the incident pressure wave on this interface sets the beam in motion, i.e. the beam acquires a velocity field $\dot{W}(x, t)$. Compatibility dictates that the wetted surface of beam and the fluid particles at its interface possess the same velocity $\dot{W}(x, t)$, provided cavitation is absent (everywhere) at the fluid and structure interface. The beam motion results in a rarefaction wave (travelling in negative $Z$-direction) of magnitude

$$
p_{R 2}(Z, x, t)=-\rho_{w} c_{w} \dot{W}\left(x, t+Z / c_{w}\right) .
$$

It is worth emphasising that the reflection of planar waves off a curved interface would render the exact formulation of the fluid pressure field too complicated to quantify. As a simplification, the reflected waves are treated as planar and propagates in the negative $Z$-direction, without affecting the pressure and particle velocity fields perpendicular to the incident angle. Thus, the net water pressure $p(Z, x, t)$ due to the incident and reflected pulses is given by

$$
\begin{aligned}
& p(Z, x, t)=p_{I}+p_{R 1}+p_{R 2}=p_{s}\left[e^{-\left(t-Z / c_{w}\right) / t_{i}}+e^{-\left(t+Z / c_{w}\right) / t_{i}}\right]- \\
& \rho_{w} c_{w} \dot{W}\left(x, t+Z / c_{w}\right) \text {. }
\end{aligned}
$$

The interface pressure (at $Z=0$ ) is, therefore,

$$
p_{\text {Int }}(x, t)=p(Z=0, x, t)=2 p_{s} e^{-t / t_{i}}-\rho_{w} c_{w} \dot{W}(x, t) .
$$

Substituting $p_{\text {Int }}(x, t)$ into Eq. 18 and rearranging gives the governing ordinary differential equations that describes the beam motion as follows:

$$
\sum_{j=1}^{n} M_{i j} \ddot{w}_{j}+\frac{\partial V}{\partial w_{i}}=B \int_{0}^{L} p_{\text {Int }}(x, t) \phi_{i}(x) \mathrm{d} x, \quad i=1,2, \ldots, n .
$$




\subsection{Limitations of the current FSI model}

The tensile term $\left(p_{R 2}\right.$ in Eq. 22) may, under certain circumstances, causes the fluid pressure to drop below zero at some point in time within the fluid domain, giving rise to a cavitation event. Following cavitation, the pressure field in the fluid is given by a superposition of two breaking fronts, one travelling towards the structure (positive $Z$ direction) and the other away (negative): generating an expanding pool of cavitated liquid [10]. Schiffer et al. [11] identified two cavitation types (or régimes) for underwater blast loading of rigid-plate with a linear backing spring that depends on the fluid conditions in the layer between the structure and the expanding cavitation zone. Type I cavitation occurs if a breaking front (travelling in the positive $Z$ direction), arrests before reaching the fluid-structure interface, reverses its motion and becomes a closing front. Type II cavitation occurs if the breaking front (travelling in positive $Z$ direction) reaches the fluid-structure interface and causes cavitation at the interface. In the present study, Type II cavitation will occur if the condition [11]

$$
p_{\text {Int }}(x, t)=0
$$

is met. Since Schiffer et al. [11] showed that a structure acquire s greater transmitted impulse in Type I than in Type II, the former would be of greater interests to a designer since the objective of this work is concerned with the limits of structural and material deformation.

In a Type I cavitation, several authors [11,12] had found that the pressure wave (rarefaction $p_{\mathrm{R} 2}$ and reflected $p_{\mathrm{R} 1}$ ) emanating from the fluid-structure interface approaches the closing front and eventually reflects back towards the fluid-structure interface as a positive pressure pulse. It was noted in [11] that this positive pressure pulse acts continuously on their rigid plate which reduces to zero after a finite duration. As a consequence, it must contribute to the impulse transmitted to the structure and in cases where the mass and stiffness of the structure are high, the maximum transmitted impulse can even exceed twice the incident impulse $I_{i}=p_{s} t_{i}$. However, detailed three-dimensional (3D) finite element simulations (to be presented later in Section 4) will show that for elasto-plastic beams deforming in any modes of deformation, the contribution of the reflection wave (from the closing front) during Type I cavitation doès not significantly affect the maximum mid-span deflection, maximum momentum, maximum transmitted impulse, maximum kinetic energy and maximum transmitted energy to the beam. On this basis, it is reasonable to neglect the influence of the reflection/wave from the closing front in the current analytical model.

\section{Finite element (FE) model}

In addition to the analytical model presented above, three-dimensional (3D) FE calculations were also performed - using the commercial software ABAQUS/ Explicit ${ }^{\circledR}$ - to model the fluid-structure interaction. Here, only the salient features of the FE model set-up are presented for the fully-clamped beam since they are described previously in Yuan et al. [1]. Details on convergence studies and validation are found there. 

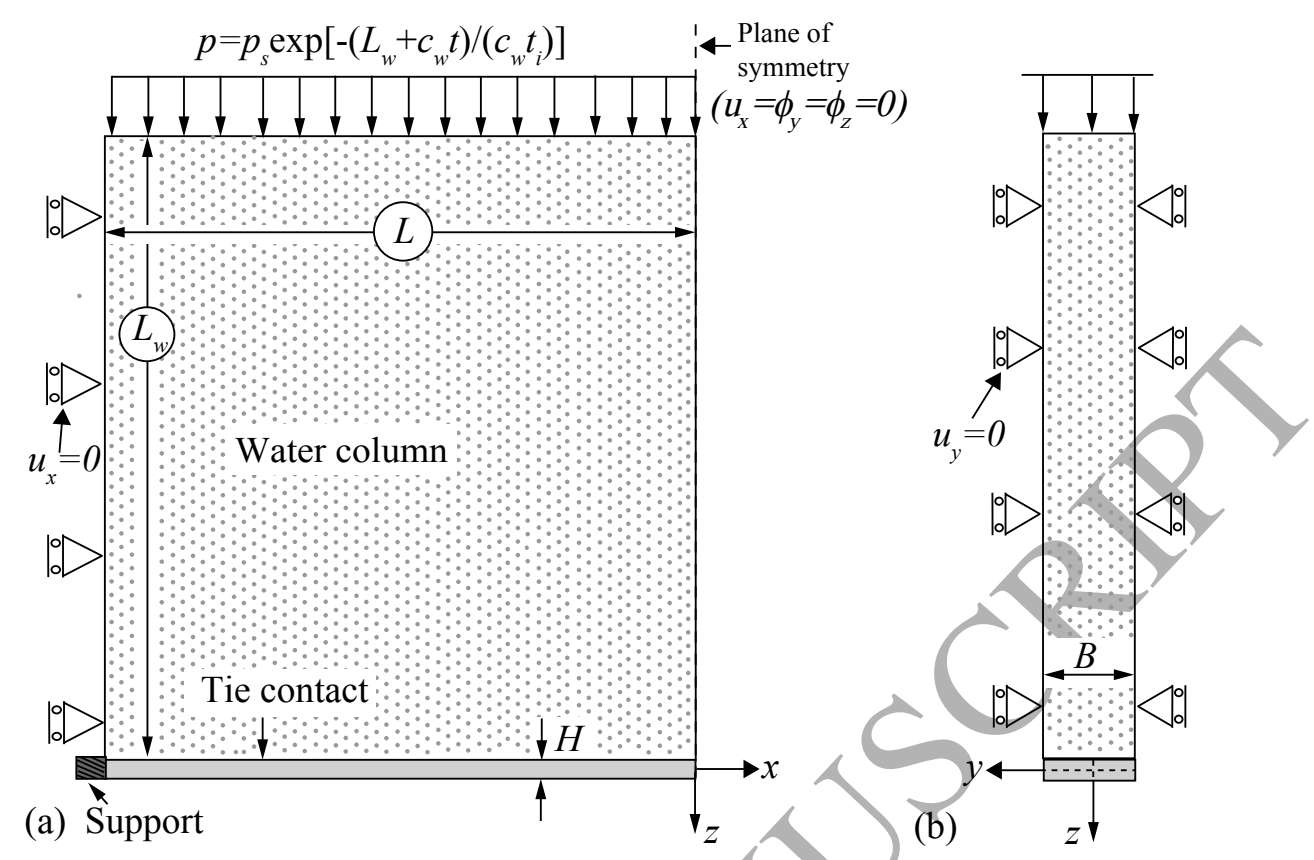

Figure 5: Schematic of clamped beam subjected to an exponentially decaying pressure pulse in FE: (a) front view and (b) side view.

\subsection{Material properties and damage model}

Material description based on the conventional $J_{2}$ flow theory is adopted to allow progressive degradation of material stiffness to be implemented in finite elements. This approach, coupled with element deletion, is widely used to model progressive damage and rupture of ductile materials [29, 30]. All the beams modelled are made of Aluminium 6061-T6 - the material properties are given in Table 2 . The progressive damage model for ductile materials in ABAQUS/Explicit is adopted here. The criterion for ductile damage initiation is given by

$$
\omega_{d}=\int \frac{d \bar{\epsilon}^{p}}{\bar{\epsilon}_{d}^{p}\left(\eta, \dot{\bar{\epsilon}}^{p}\right)}=1
$$

where $\omega_{d}$ is a state variable that increases monotonically with the equivalent plastic strain. Here, the equivalent plastic strain $\bar{\epsilon}_{d}^{p}$ at the onset of ductile damage is a function of stress triaxiality $\eta$ and plastic strain rate $\dot{\bar{\epsilon}}^{p}[31]$. When Eq. 27 is met, the damage variable $D$ would increase according to [16]

$$
\dot{D}=\frac{L_{e} \dot{\bar{\epsilon}}^{p}}{\bar{u}_{f}^{p}}
$$

where $\bar{u}_{f}^{p}$ is the effective plastic displacement at failure and $L_{e}=7.83 \times 10^{-4} \mathrm{~m}$ is the characteristic length of the first-order element used in the FE model - this is identical to that in [1]. Any element whose stiffness is fully degraded, i.e. $D=1$, is deleted from the mesh. The two parameters needed to implement a ductile damage model are the damage 
strain $\bar{\epsilon}_{d}^{p}=0.8$ and the failure displacement $\bar{u}_{f}^{p}=1.1 \times 10^{-3} \mathrm{~m}$; both were obtained through calibration to the experimental data by Menkes and Opat [19].

The water is modelled as an acoustic medium with density $\rho_{w}=1000 \mathrm{~kg} / \mathrm{m}^{3}$, wave speed $c_{w}=1498 \mathrm{~m} / \mathrm{s}$ and bulk modulus $E_{w}=2.244 \mathrm{GPa}$. It can be treated as linear elastic under compression, with zero tensile strength and zero shear modulus. A Mie-Gruneisen equation of state with a linear Hugoniot relation is used to model the linear elastic ratio between applied pressure $p(Z, t)$ and volumetric strain $\epsilon_{V}$ given by

$$
p(Z, t)=\left\{\begin{array}{l}
-E_{w} \epsilon_{V}=-c_{w}^{2} \rho_{w} \epsilon_{V}, \quad \epsilon_{V}<0 \\
0, \quad \epsilon_{V} \geq 0
\end{array} .\right.
$$

Note that when $\epsilon_{V} \geq 0$, the pressure becomes zero and this leads to an onset of cavitation $[3,4,32]$.

\subsection{Mesh, loading and boundary conditions}
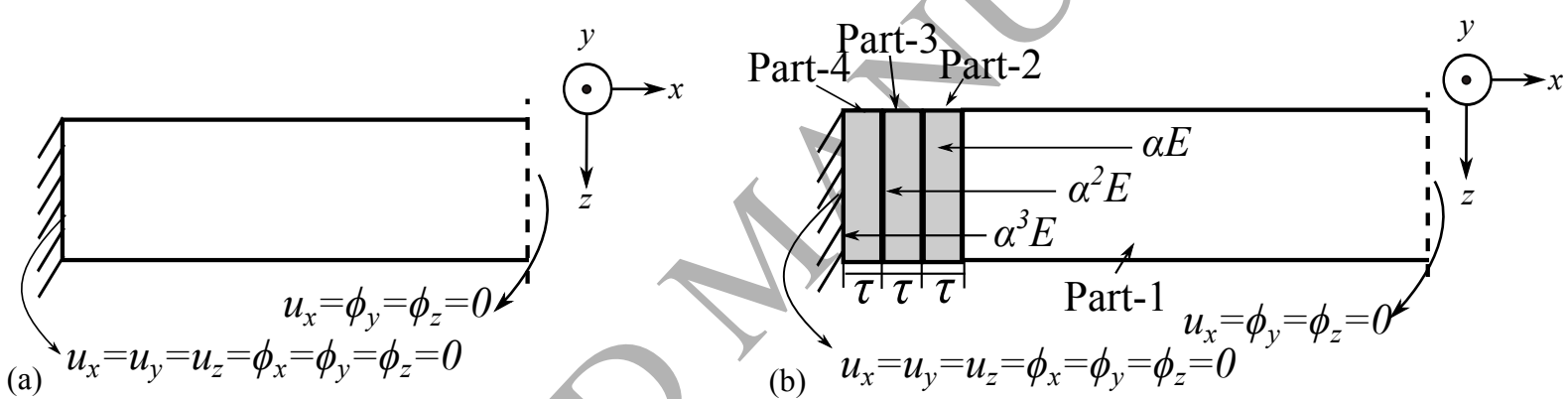

Figure 6: Schematic of boundary, or support, conditions (BCs) in the FE simulations: (a) standard fully clamped BC and (b) modified BC. $u$ and $\phi$ denote displacement and rotation degree of freedom, respectively.

The FE model, shown schematically in Fig 5, consists of a water column $L_{w} \times L \times B$ above the elasto-plastic beam. Only one-half of the fully-clamped beam and water column are modelled since reflective symmetry exists on the plane bounded by $x=0,-B / 2 \leq y \leq B / 2$ and $-H / 2-L_{w} \leq z \leq H / 2$. Figure 6 a depicts the standard displacement boundary conditions that would need to be imposed for a fully-clamped boundary condition (BC). It has been shown in [1] that the local equivalent plastic strain in the beam abutting the supports does not converge with repeated mesh refinement. In order to accurately model progressive ductile fracture /rupture at the supports, a modified BC given in Fig 6b is adopted. Yuan et al. [1] showed that both the standard and modified BCs predict similar beam deflection profiles, but only the latter gives a converged equivalent plastic strain at the boundary/support. A similar procedure was also adopted by Yuan and Tan [33] to model the impulsive response of rectangular plates numerically. For the modified BC, three additional parts (labelled 2-4) are added to the end of the original solid beam (part-1) to form an extended boundary shown schematically in Fig 6b. Note that the standard fully-clamped $\mathrm{BC}$ of Fig $6 \mathrm{a}$ is imposed on part 4. All the additional parts have equal width $\tau$ and identical 
material properties as the solid beam, apart from a gradation of their elastic modulus $E$, by a factor $\alpha$. The parameters $\tau=H / 6$ and $\alpha=10$ were obtained by calibration to the experimental data of [19] - the details are provided in Yuan et al. [1].

Horizontal displacement $u_{x}$ for the left boundary of the water column $\left(u_{x}=0\right.$ at $x=-L$, $\left.-B / 2 \leq y \leq B / 2,-H / 2-L_{w} \leq z \leq-H / 2\right)$ is constrained as does the vertical displacement $u_{y}$ on the front $\left(u_{y}=0\right.$ at $\left.-L \leq x \leq 0, y=B / 2,-H / 2-L_{w} \leq z \leq-H / 2\right)$ and back $\left(u_{y}=0\right.$ at $\left.-L \leq x \leq 0, y=-B / 2,-H / 2-L_{w} \leq z \leq-H / 2\right)$ boundary of the water column - see Fig 5. An exponentially decaying pressure pulse, given by Eq 20, is applied to the top of the water column. Tie constraints are applied between the wetted edge of the beam and fluid.

The half-beam modelled has a dimension of $0.06 \mathrm{~m}(L) \times 0.01 \mathrm{~m}(H) \times 0.01 \mathrm{~m}(B)$. The length of water column $L_{w}$ is sufficient long to ensure the reflected wave from the top boundary does not reach the structure over the duration of the calculation $[3,4] . L_{w} / c_{w} t_{i}=3$ is used to ensure that the water column is semi-infinite. Both the beam and water column are discretised using the 8-noded solid elements (C3D8R) with reduced integration and hour-glass control. In order to capture necking localisation, progressive damage and ductile fracture with acceptable fidelity, twelve elements are used through the beam thickness $(H)$. Results of convergence studies have shown this to be sufficient. All C3D8R elements - used to discretise the water column and beam - are cubic in size with a dimension of $7.83 \times 10^{-4} \mathrm{~m}$; consequently, the nodes of the water and solid meshes are coincident at the fluid-structure interface.

\section{Comparison of analytical and FE predictions}

To assess the fidelity of the analytical model, its predictions for the average interface pressure, mid-span deflection, impulse and energy transfer will now be compared to predictions by the FE model. Appendix A gives all the energy expressions for the three phases of deformation and a flow chart on the numerical implementation of the analytical model is presented in Section 2. The temporal history of the transmitted impulse per unit area $I^{T}(t)$, momentum per unit area $I^{K}(t)$, transmitted energy per unit area $E^{T}(t)$ and kinetic energy per unit area $E^{K}(t)$ of the beam are defined, respectively, as follows:

$$
\begin{aligned}
I^{T}(t) & =\int_{0}^{t} \tilde{p}_{\text {Int }}(t) \mathrm{d} t, \quad I^{K}(t)=\frac{\rho H}{L} \int_{0}^{L} \dot{W}(x, t) \mathrm{d} x, \\
E^{T}(t) & =\frac{1}{B L} \int_{0}^{L} p_{\text {Int }}(x, t) W(x, t) \mathrm{d} x, \quad E^{K}(t)=\frac{\rho H}{2 L^{2}} \int_{0}^{L} \dot{W}^{2}(x, t) \mathrm{d} x .
\end{aligned}
$$

Note that the average interface pressure is given by

$$
\tilde{p}_{\text {Int }}(t)=\frac{1}{L} \int_{0}^{L} p_{\text {Int }}(x, t) \mathrm{d} x .
$$


Time $t$ is measured from the instant when the incident pressure wave arrives at the fluid and structure interface. The impulse and energy components in Eq. 30 are normalised by the incident impulse per unit area $I_{i}$ and incident energy per unit area $E_{i}$ given by [2]

$$
I_{i}=p_{s} t_{i} \quad \text { and } \quad E_{i}=p_{s}^{2} t_{i} / \rho_{w} c_{w} .
$$

Unless otherwise specified, a time constant of $t_{i}=0.2 \mathrm{~ms}$ - corresponding to $16 \mathrm{~kg}$ of TNT at a $1.4 \mathrm{~m}$ standoff distance according to the scaling law by Swisdak [34] - was used in all calculations; consequently, variations of FSI index $\beta_{w}$ is achieved by altering the beam thickness. It is worth emphasising that in both analytical and FE model, if the structural system fails (i.e. complete detachment occurs) before all the initial kinetic energy is expended, then the beam member would have a residual kinetic energy at the point of severance. Parts of this are absorbed through further plastic deformation as the beam member continues to deform until it reaches a rigid permanent set whilst the remaining as translational kinetic energy. Since fluid-structure interaction in the 'pre-detachment' régime is of interests, the energy (and impulse) transferred 'post-detachment' by the blast wave is not considered, or characterised, here.

Figures $7 \mathrm{a}, 7 \mathrm{~b}$ and $7 \mathrm{c}$ compare the analytical and FE predictions for beams that deform in mode I, II and III, respectively. Pressure contour plots predicted by FE (to be presented later in Fig 8) confirm that a Type $I$ cavitation event (i.e. the breaking front travelling in the positive $Z$-direction, always arrests before it reaches the fluid-structure interface and inverts its motion as a closing front) occurs in all the cases shown in Figs $7 \mathrm{a}, 7 \mathrm{~b}$ and $7 \mathrm{c}$ at $t_{c} / t_{i}=0.53, t_{c} / t_{i}=0.41$ and $t_{c} / t_{i}=0.36$, respectively. For beams deforming in mode I, Figure $7 \mathrm{a}$ shows that their non-dimensional average interface pressure $\tilde{p}_{\text {Int }} / p_{s}$ initially decreases before attaining a peak value at time $t=t_{3}$ (when the beam reaches its maximum mid-span deflection); this is followed by a monotonic reduction in interface pressure. Beyond $t>t_{3}$, the FE model predicts that $\tilde{p}_{\text {Int }} / p_{s}$ remains positive - this is in agreement with [11]. The analytical model under-predicts the maximum transmitted impulse in mode I by up to $12.7 \%$ since it neglects the additional loading from the reflected wave arising from the closing front. Complete detachment occurs shortly after cavitation in Figs 7b and 7c which explains why it is acceptable to neglect the effects of the reflected wave from the closing front in the subsequent structural response. In general, the predicted interface pressure, mid-span deflection, impulse and energy exchange are in good agreement with those from FE for all three modes of deformation.

The impulse and energy transferred to the beam through FSI, also plotted in Fig 7, can be succinctly summarised as follows: (1) In mode I, the average interface pressure beyond $t>t_{3}$ does not further contribute to the transmitted energy $E^{T}$. This is because the beam now responds in an elastic manner with deflection that decreases slightly before reaching permanent set; (2) In modes II and III, both the transmitted impulse $I^{T}$ and transmitted energy $E^{T}$ reach a maximum at $t=t_{3}$ following complete detachment from the supports; (3) The maximum momentum $I^{K}$ and kinetic energy $E^{K}$ are reached before the onset of cavitation at $t=t_{c}$ for all three modes, this also agrees with analytical predictions in [11]; (4) The maximum transmitted impulse is significantly higher in mode I than in modes II 


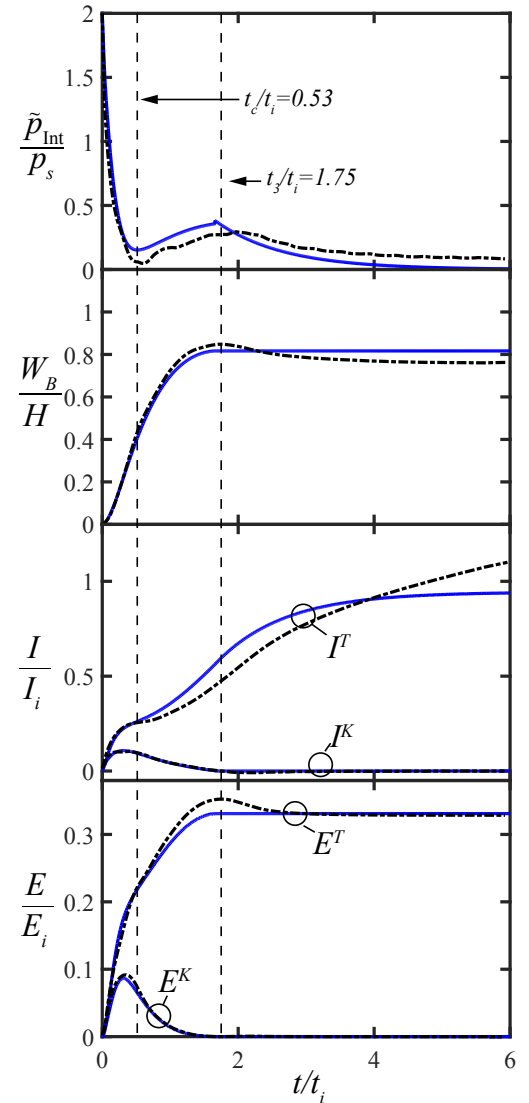

(a) Mode I

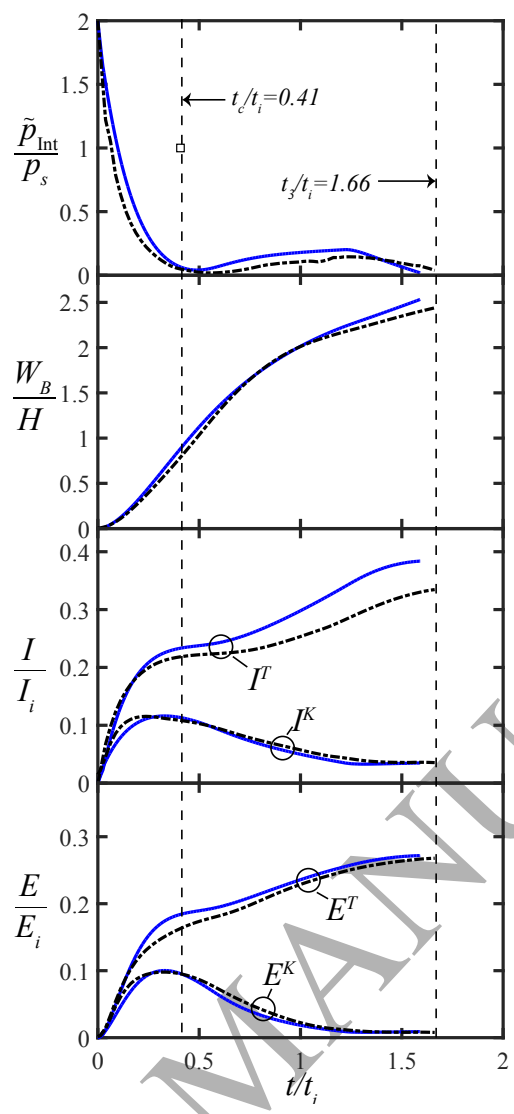

(b) Mode II

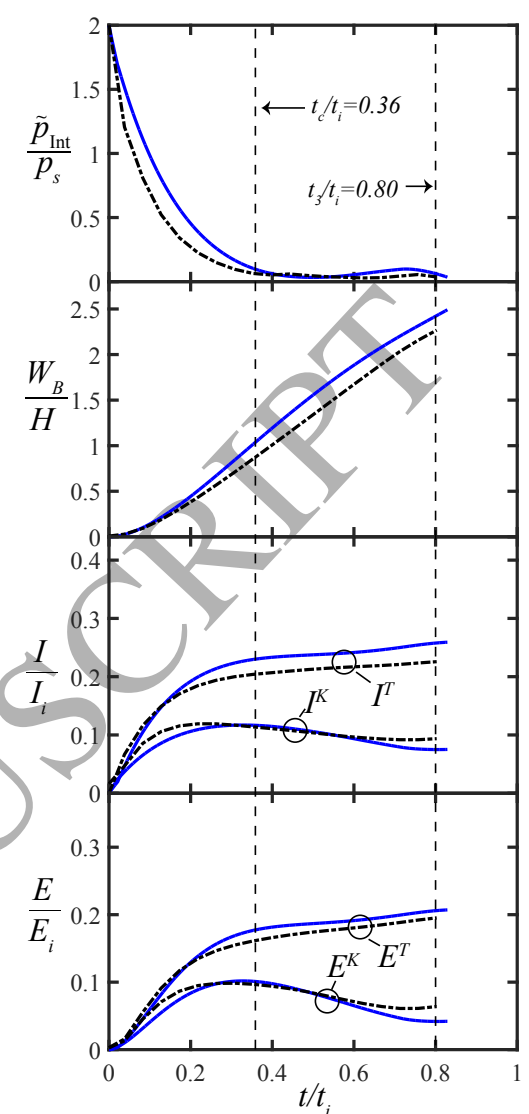

(c) Mode III

Figure 7: Analytical and FE predictions of the non-dimensional interface pressure, mid-span deflection, impulse transfer and energy exchange for $0.06 \mathrm{~m}(L) \times 0.01 \mathrm{~m}(H) \times 0.01 \mathrm{~m}(B)\left(L / H=6\right.$ and $\left.\beta_{w}=10.9\right)$ beam deforming in different modes subjected to three different exponentially decaying pressure pulses of identical time constant $t_{i}=0.2 \mathrm{~ms}$ and a peak pressure of (a) $p_{s}=30 \mathrm{MPa}$, (b) $p_{s}=80 \mathrm{MPa}$ and (c) $p_{s}=110 \mathrm{MPa}$. - current analytical predictions; -.- current FE predictions. $t_{c}$ and $t_{3}$ denote the time when cavitation first occurs and when the beam reaches its maximum transverse deflection, respectively.

and III because a considerable amount of impulse is transmitted to the beam during elastic rebound; and, (5) The maximum transmitted impulse and energy are higher in mode II than III since $t_{3}$ for mode III is smaller than mode II.

To gain an insight into the cavitation event, pressure contour maps predicted by detailed 3D FE simulations are plotted in Fig 8 at five selected times (corresponding to the elasto-plastic beam and loading parameters of Fig 7). Here, only the results for a third of the 'water-tank', i.e. $L_{w} / 3$, is plotted. It is worth emphasising that cavitation first occurs at $t_{c} / t_{i}=0.53$ (for mode I), $t_{c} / t_{i}=0.41$ (for mode II) and $t_{c} / t_{i}=0.36$ (for mode III). This is caused by the tensile wave (Eq. 22) which is generated as a consequence of the rapid motion of the beam and is reminiscent of the experimental observations made in [12] for circular plates. All the cases shown in Fig 8 correspond to a Type $I$ cavitation event since the breaking 

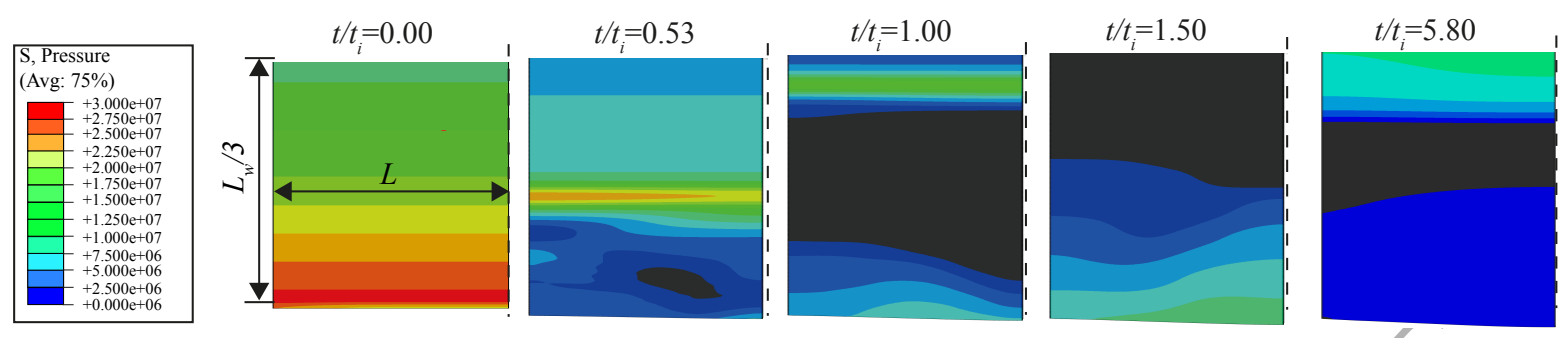

(a) Mode I
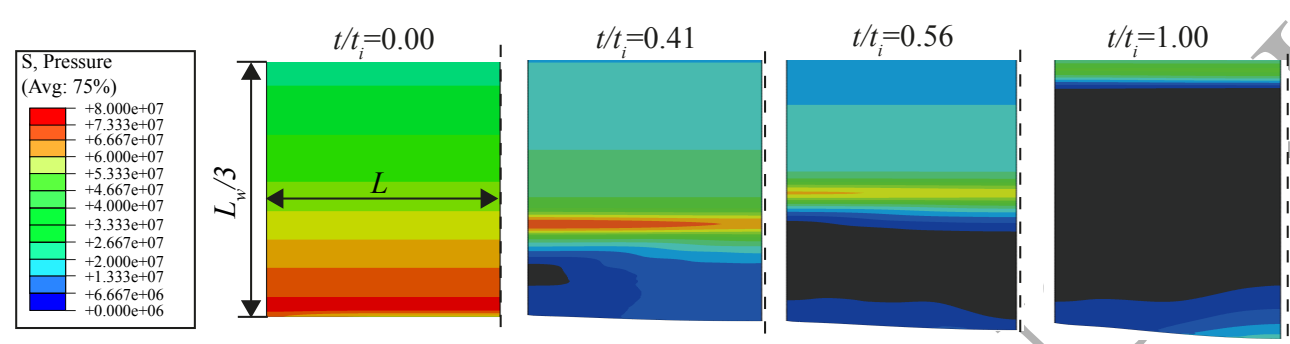

$t / t=1.50$

(b) Mode II
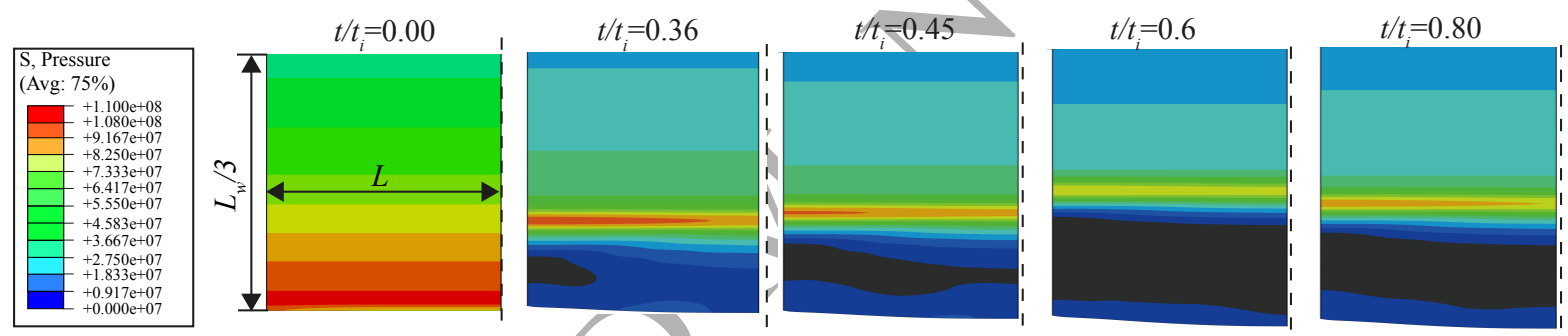

(c) Mode III

Figure 8: FE predictions of fluid pressure field at five selected times corresponding to the cases $(L / H=6$ and $\beta_{w}=10.9$ ) shown in Figs 7a, $7 \mathrm{~b}$ and 7c, respectively. Black denotes cavitated water. Note that only a third of the water tank that was simulated had been plotted here.

front arrests and inyerts its motion as a closing front. Note that a closing front (this refers to the bottom edge of the cavitated region) has already formed at $t / t_{i}=1.00,1.00$ and 0.8 in Figs $8 \mathrm{a}, 8 \mathrm{~b}$ and $8 \mathrm{c}$, respectively. It is worth highlighting why the cavitated region at $t / t_{i}=5.8$ appears closer to the fluid-structure interface compared to $t / t_{i}=1.5$ in Fig 8 a. At $t / t_{i}=1.0$, the entire cavitated region is in the midst of propagating towards the top of the water-tank whereupon, after reflection, it would reverse its motion towards the structure. What was shown for $t / t_{i}=5.8$ is the reflected cavitated region from the top boundary which is now propagating towards the structure - this is why the bottom boundary of the cavitated region appears closer to the structure at $t / t_{i}=5.8$ compared to, say, at $t / t_{i}=1.5$ in Fig 8 a.

Predictions by FE and the current analytical model will now be compared for a range of incident impulses where the primary focus will be on the maximum mid-span deflection, transmitted impulse and energy. The maximum non-dimensional transmitted impulse $\bar{I}^{T}$, 


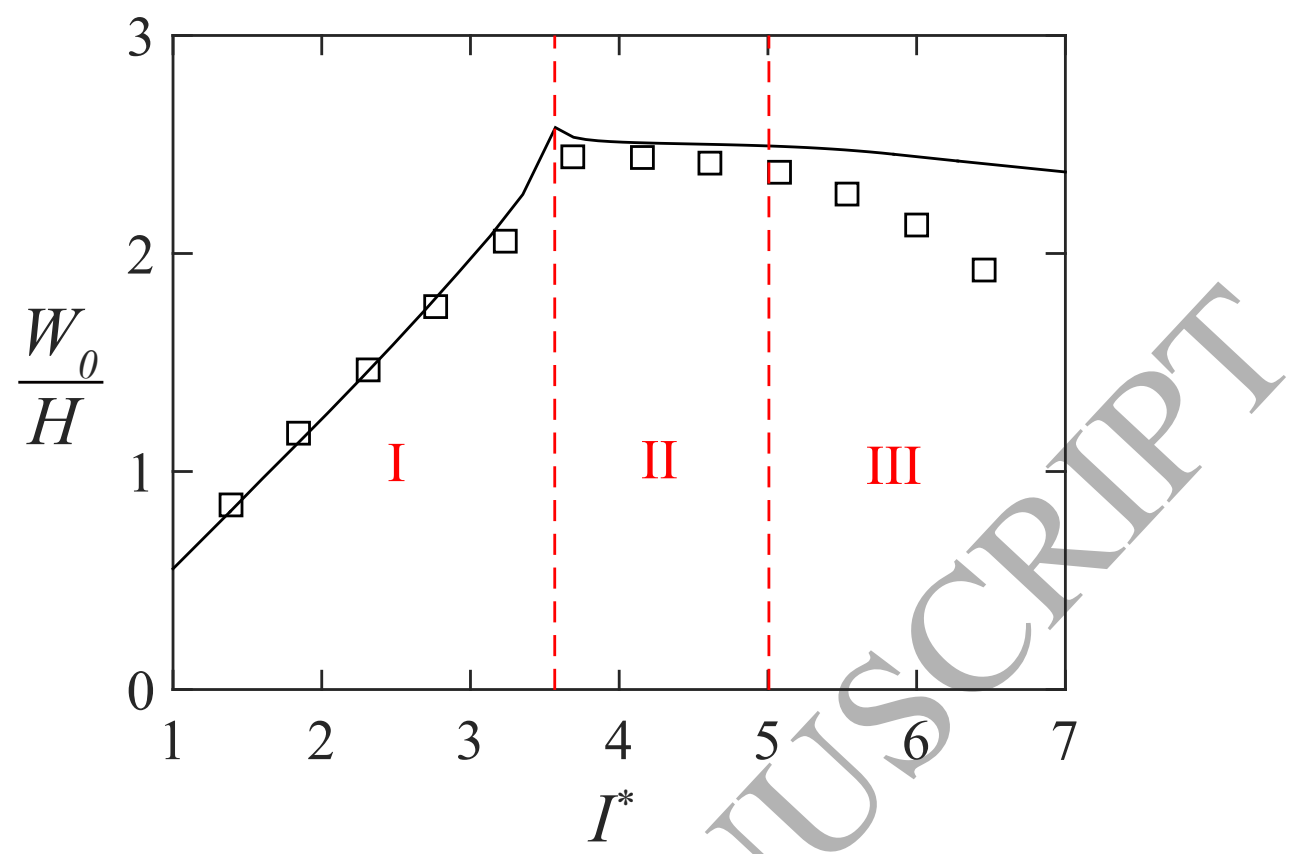

Figure 9: Variations of the non-dimensional mid-span deflection $W_{0} / H$ with non-dimensional impulse $I^{*}$ for elasto-plastic beam with $L / H=6$ and $\beta_{w}=10.9$. I, II and II $Y$ indicate the three distinct damage modes predicted by the current analytical model. — current analytical model; $\square$ current FE predictions.

momentum $\bar{I}^{K}$, transmitted energy $\bar{E}^{T}$ and kinetic energy $\bar{E}^{K}$ of the elasto-plastic beam are defined as follows:

$$
\begin{aligned}
& \bar{I}^{T}=\max _{0 \leq t<\infty}\left[I^{T}(t) / I_{i}\right], \quad \bar{I}^{K}=\max _{0 \leq t<\infty}\left[I^{K}(t) / I_{i}\right], \\
& \bar{E}^{T}=\max _{0 \leq t<\infty}\left[E^{T}(t) / E_{i}\right], \quad \bar{E}^{K}=\max _{0 \leq t<\infty}\left[E^{K}(t) / E_{i}\right] .
\end{aligned}
$$

Figure 9 shows an excellent agreement for the maximum mid-span deflection $W_{0} / H$, at either the point of cessation of motion or failure (if complete detachment from the supports had occurred) for modes I and II. The over-prediction of $\Delta W_{0} / H$ in mode III with $I^{*}(\triangleq$ $\left.2 p_{s} t_{i} / H \sqrt{\sigma_{Y} \rho}\right)$ is because our analytical model adopts a constant hinge length of $l=2 H$ the same assumption was also made in [17], [23] and [18] - as opposed to the approach by Shen and Jones [20] where a new hinge length is re-calibrated for each data point. Both the current $\mathrm{FE}$ and analytical models correctly predict a reduction in $W_{0} / H$ with increasing $I^{*}$ in modes II and III - this trend is also observed in [1].

Figure 10 compares the non-dimensional impulse $\left(\bar{I}^{T}\right.$ and $\left.\bar{I}^{K}\right)$ and energy $\left(\bar{E}^{T}\right.$ and $\left.\bar{E}^{K}\right)$ terms with the corresponding FE predictions. In general, the analytical predictions agree well with its FE counterpart despite the under-prediction of $\bar{I}^{T}$ - by up to a maximum 


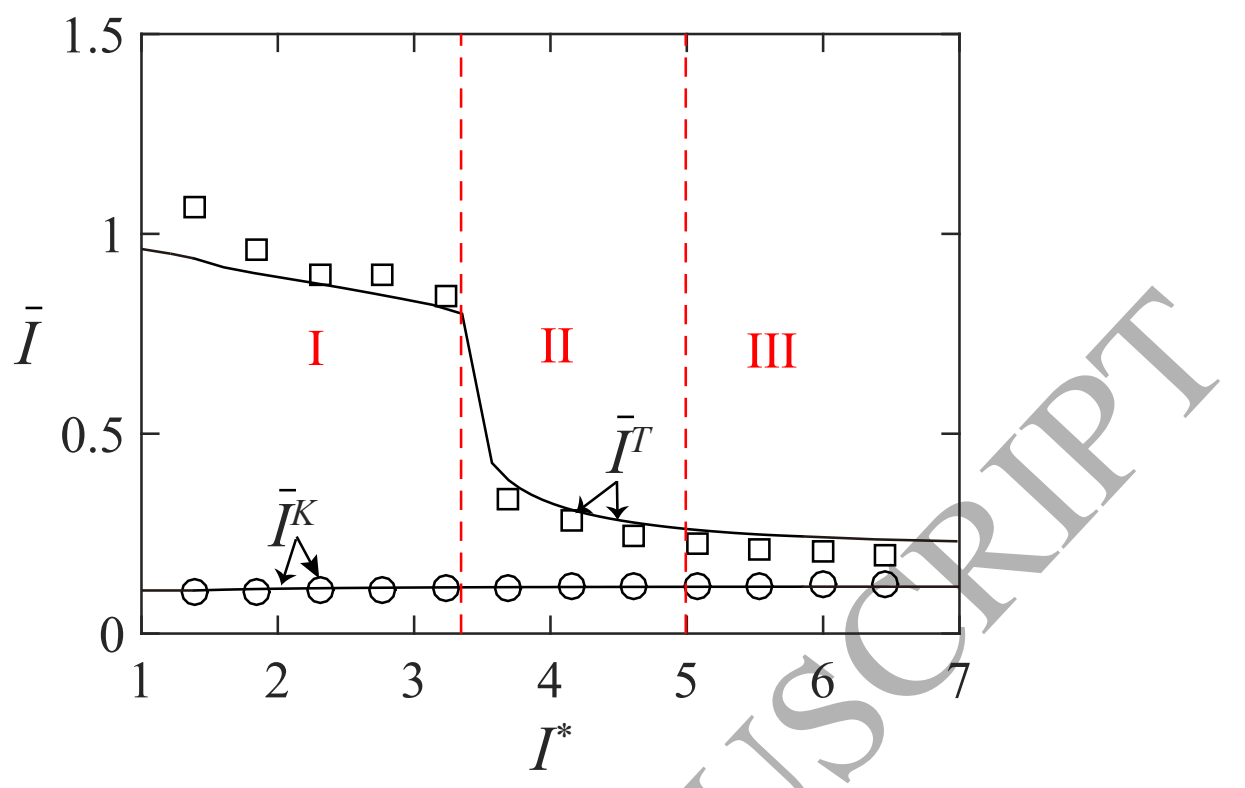

(a)

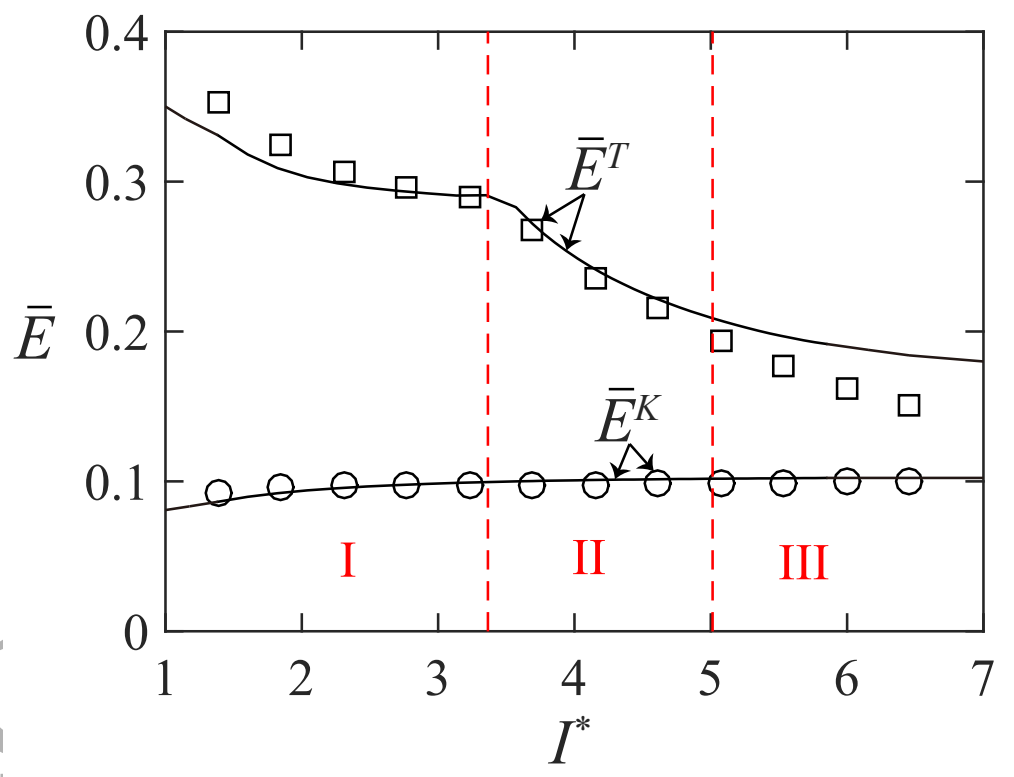

(b)

Figure 10: Variations of the non-dimensional maximum impulse and energy against non-dimensional impulse $I^{*}$ for elasto-plastic beam with $L / H=6$ and $\beta_{w}=10.9$. I, II and III indicate the three distinct damage modes predicted by the current analytical model. — current analytical model; $\square$ and $\mathrm{O}$ current FE predictions.

of $12.7 \%$ - in mode I. This discrepancy arises because our analytical model neglects the additional impulse transmitted by the reflection wave from the closing front. 
and

In a Type I cavitation event (i.e. cavitation does not occur first at the fluid-structure interface), the maximum non-dimensional transmitted impulse $\bar{I}^{T}$ can be shown to be related to the average maximum transverse deflection, as well as the time $\left(t_{3}\right)$ for complete detachment to occur; note that $t_{3}=1.66 t_{i}$ and $t_{3}=0.8 t_{i}$ in Figs $7 \mathrm{~b}$ and $7 \mathrm{c}$, respectively. Substituting Eqs 30 and 31 into Eq. 33, and re-arranging, gives

$$
\bar{I}^{T}=\left(1 / I_{i}\right) \int_{0}^{\infty} \tilde{p}_{\text {Int }}(t) \mathrm{d} t, \quad \text { if mode I }
$$

$$
\bar{I}^{T}=\left(1 / I_{i}\right) \int_{0}^{t_{3}} \tilde{p}_{\text {Int }}(t) \mathrm{d} t, \quad \text { if mode II and III. }
$$

and

Using Eqs. 15 and 24 in Eqs. 34 and 35, respectively, leads to an expression for the nondimensional impulse $\bar{I}^{T}$ as follows:

$$
\bar{I}^{T}=2-\frac{\rho_{w} c_{w}}{I_{i}} \int_{0}^{t_{3}} \int_{0}^{L}\left[\sum_{i=1}^{n} \frac{\phi_{i}(x) \dot{w}_{i}(t)}{L}\right] \mathrm{d} x \mathrm{~d} t-\frac{\rho_{w} c_{w}}{I_{i}} \int_{t_{3}}^{\infty} \int_{0}^{L}\left[\sum_{i=1}^{n} \frac{\phi_{i}(x) \dot{w}_{i}(t)}{L}\right] \mathrm{d} x \mathrm{~d} t, \quad \text { if mode I }
$$

$$
\bar{I}^{T}=2-2 \mathrm{e}^{-t_{3} / t_{i}}-\frac{\rho_{w} c_{w}}{I_{i}} \int_{0}^{t_{3}} \int_{0}^{L}\left[\sum_{i=1}^{n} \frac{\phi_{i}(x) \dot{w}_{i}(t)}{L}\right] \mathrm{d} x \mathrm{~d} t, \quad \text { if mode II or III. }
$$

Since the mid-span velocity of the beam is negligibly small during elastic rebound ( see Fig 7a for example), it is reasonable to neglect the second term in Eq. 36 which simplifies to

$$
\bar{I}^{T}=2-\frac{\rho_{w} c_{w}}{I_{h}} \int_{0}^{t_{3}} \int_{0}^{L}\left[\sum_{i=1}^{n} \frac{\phi_{i}(x) \dot{w}_{i}(t)}{L}\right] \mathrm{d} x \mathrm{~d} t, \quad \text { if mode I. }
$$

Thus, the maximum non-dimensional impulse transmitted to the beam is related to the different mechanisms through which alleviation of the transmitted impulse is achieved as follows:

$$
\bar{I}^{T}=\bar{I}_{1}^{T}-\bar{I}_{2}^{T}-\bar{I}_{3}^{T}
$$

where $\bar{I}_{1}^{T}=2$ - this is a reference (or maximum) value - denotes the maximum nondimensional impulse transmitted to a rigid beam of infinite mass; $\bar{I}_{2}^{T}=0$ (if mode I) or $\bar{I}_{2}^{T}=2 \mathrm{e}^{-t_{3} / t_{i}}$ (if mode II or mode III) refers to the reduction of transmitted impulse as a consequence of boundary failure or detachment; and $\bar{I}_{3}^{T}=\rho_{w} c_{w} \hat{W} / I_{i}$ quantifies the alleviation of transmitted impulse due to both non-linear material deformation and support compliance, where $\hat{W}$ is the average transverse deflection of the beam given by

$$
\hat{W}=\int_{0}^{t_{3}} \int_{0}^{L}\left[\sum_{i=1}^{n} \frac{\phi_{i}(x) \dot{w}_{i}(t)}{L}\right] \mathrm{d} x \mathrm{~d} t
$$

Equation 39 suggests that early boundary failure (a smaller $t_{3}$ leads to higher $\bar{I}_{2}^{T}$ which is evident by comparing Fig 7c to 7b) and/or large material deformation (a higher $\bar{I}_{3}^{T}$ ) would 
contribute to a significant reduction in the maximum non-dimensional impulse $\left(\bar{I}^{T}\right)$ that is transmitted.

Figure 10a shows that $\bar{I}^{T}$ decreases only slightly with $I^{*}$ in mode I. This is because the maximum mid-span deflection $W_{0}$ - which is related to the average transverse deflection $\hat{W}$ - increases linearly with $I^{*}$ (or $I_{i}$ ) in mode I (see Fig 9) which leads to a higher $\bar{I}_{3}^{T}$ and a consequential reduction in $\bar{I}^{T}$. A sharp decrease in $\bar{I}^{T}$ follows the transition from mode $\mathrm{I} \rightarrow \mathrm{II}$ because of boundary failure; a direct consequence of a jump in $\bar{I}_{2}^{T}$ from zero to a finite value in Eq 39). Figure 10a shows a monotonic reduction in $\bar{I}^{T}$ with $I^{*}$ in modes II and $I U$. This is because a higher $I^{*}$ leads to premature boundary failure, resulting in a greater reduction of the transmitted impulse ( $\bar{I}_{2}^{T}$ in Eq. 39$)$, see Figs 7b and 7c. It is notable that a higher $I^{*}$ also leads to greater transmitted impulse $\bar{I}^{T}$; this is a direct consequence of a lower $\bar{I}_{3}^{T}$ since $W_{0}$ in modes II and III reduces monotonically with $I^{*}$ as shown in Fig 9. The monotonic reduction of $\bar{I}^{T}$ in modes II and III indicates that $\bar{I}_{2}^{T}$ could be more dominant than $\bar{I}_{3}^{T}$ when complete detachment occurs at the support. Figure 10 also shows that the maximum transmitted energy $\bar{E}^{T}$ reduces monotonically with $I^{*}$ in modes II and III; again, this is due to pre-mature detachment of the beam from its supports. As alluded to earlier, the impulse and energy transmitted 'post-detachment' are not considered. This curtails the amount of impulse and energy transmitted to the elasto-plastic beams if the incident pressure pulse is sufficiently intense.

It is interesting to note that both $\bar{I}^{K}$ and $\bar{E}^{K}$ appears relatively insensitive to $I^{*}$. This is because the elasto-plastic beams, regardless of their deformation mode, always acquires the maximum $\bar{I}^{K}$ well before the onset of cavitation in the fluid; the same was also reported in [11]. The maximum $\bar{I}^{K}$ is already attained when the travelling plastic hinge had only just started to emanate from each end of the support; hence, the central portion of the beam is reminiscent of a rigid, free-standing beam. Schiffer and Tagarielli [12] also reported a similar 'central rigid portion' for their fully-clamped circular composite plate in an underwater blast. Recall that for rigid free-standing structures, the maximum non-dimensional momentum acquired is independent of load intensity since it is only a function of FSI index [2]. Because of the above, the maximum non-dimensional transmitted momentum $\bar{I}^{K}$ also appears independent of blast impulse; similarly, for $\bar{E}^{K}$.

\section{Effects of FSI on 'deformable supported' versus 'rigid free-standing' beams}

Parametric studies will now be carried out, using the coupled fluid-beam analytical model, to evaluate the sensitivity of the maximum impulse $\left(\bar{I}^{T}\right)$ and energy $\left(\bar{E}^{T}\right)$ transfer to aspect ratio $L / H$ and $\beta_{w}\left(\triangleq \rho_{w} c_{w} t_{i} / \rho H\right.$ - Taylor's FSI index $)$. Results from rigid free-standing beams of an equivalent mass per unit area are also included for comparison where the maximum transmitted impulse and energy - superscript $F$ denotes free-standing - are, respectively, given by [2]

$$
\bar{I}^{F}=2 \beta_{w}^{-\left(\beta_{w} / \beta_{w}-1\right)}
$$


and

$$
\bar{E}^{F}=2 \frac{\left(1-e^{-\beta_{w}}\right)^{2}}{\beta_{w}} .
$$

In the parametric studies to be presented, it is worth noting the following: (1) the nondimensional maximum transmitted impulse $\bar{I}^{F}$ and energy $\bar{E}^{F}$ of rigid free-standing beams are independent of peak incident pressure $p_{s}$ and aspect ratio $L / H$ but depends only on $\beta_{w}$; (2) both the 'elasto-plastic' and 'rigid, free-standing' beams have identical mass per unit area given by $\beta_{w}$; and, (3) cavitation always occurs at the fluid and structure interface for free-standing beams at time $t_{c}=t_{i} \ln \left(\beta_{w}\right) /\left(\beta_{w}-1\right)$ whilst it does not for the elasto-plastic beams (Type $I$ cavitation); hence, the fluid-structure interaction time for an elasto-plastic beam is always considerably longer compared to its free-standing counterpart of the same mass per unit area.

\subsection{Mode $I$}

The solid blue lines in Figs 11a and 12a denote the non-dimensional maximum transmitted impulse $\left(\bar{I}^{T}\right.$ and $\left.\bar{I}^{F}\right)$ and the broken black lines denote the non-dimensional maximum transmitted energy $\left(\bar{E}^{T}\right.$ and $\left.\bar{E}^{F}\right)$. A peak incident overpressure of $p_{s}=15 \mathrm{MPa}$ is used throughout so that the elasto-plastic beams always deform in mode I. Figure 11a plots the variation of the non-dimensional maximum impulse and energy terms as a function of $L / H$ (for a constant $\beta_{w}=21.7$ ). For elastó-plastic beams, $\bar{I}^{T}$ reduces monotonically with $L / H$ while $\bar{E}^{T}$ does not. The reason is evident by comparing two beams of different $L / H$, denoted by (1) and (2) in Fig 11b. The beam with a higher $L / H$, i.e. beam (2), has less average interface pressure $\tilde{p}_{\text {Int }} / p_{s}$ but higher mid-span deflection $W_{B}$, leading to a smaller $\bar{I}^{T}$ and non-monotonic decreasing $\bar{E}^{T}$. Figure 12a plots the maximum non-dimensional impulse and energy to FSI index $\beta_{w}$ with a constant $L / H=10$. It shows that $\bar{I}^{T}$ increases monotonically with increasing $\beta_{w}$ (less mass per unit area) but $\bar{E}^{T}$ does not. Again, the reason is that the average interface pressure $\tilde{p}_{\text {Int }} / p_{s}$ is higher but the mid-span deflection $W_{B}$ is lower for beam with a higher $\beta_{w}$ (less mass per unit area) - compare beam (3) to (2) in Fig 12b.

According to Eq. 39, increasing maximum mid-span deflection would lead to a greater reduction of $\bar{I}^{T}$, i.e. by comparing the maximum mid-span deflection between beam (1) and (2) in Fig $11 \mathrm{~b}$ or between beam (2) and (3) in Fig 12b. Notice that this finding is independent of beam's geometry (i.e. two beams can have different $L / H$ and $\beta_{w}$ ), when comparing beam (1) (in Fig 11) to (3) (in Fig 12). The above finding is potentially useful for designers as the maximum mid-span deflection (in mode I) could - alone - be used as an indicator of the alleviation of the impulse imparted to the structure.

Even though the beneficial effects of FSI, in reducing the maximum transmitted impulse for a given incident pressure pulse, is clearly evident for both the deformable (and supported) and rigid (and unsupported) beams, the mechanisms by which this reduction is achieved are clearly different. In the former, its is due to increased elasto-plastic deformation whereas, in the latter, it is due to early cavitation (a reduction in the beam's mass per unit area). We have previously shown in Eq. 39; that the maximum impulse transmitted to a deformable 

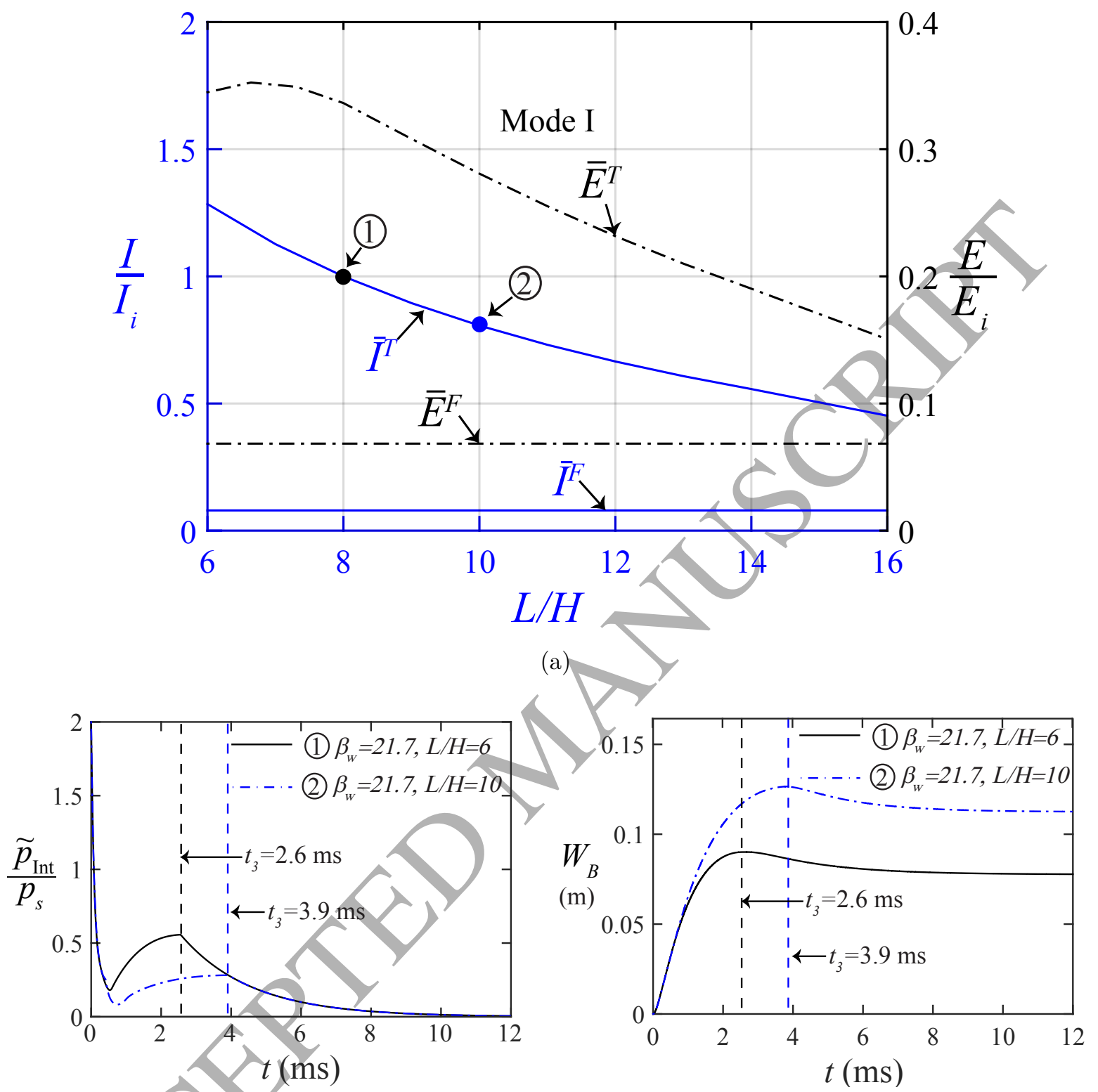

(b)

Figure 11: a) Analytical prediction of non-dimensional maximum impulse (— corresponds to $\bar{I}^{T}$ and $\bar{I}^{F}$ ) and energy (-. corresponds to $\bar{E}^{T}$ and $\bar{E}^{F}$ ) for elasto-plastic beams of identical cross-section, $H=B=0.005$ $\mathrm{m}\left(\beta_{w}=21.7\right)$, deforming in mode $\mathrm{I}$; and (b) Comparison of the normalised temporal average interface pressure $\tilde{p}_{\text {Int }}(t) / p_{s}$ and temporal mid-span deflection $W_{B}(t)$ for beams (1) and (2) in Fig 11a. Beam (1) is $0.04 \mathrm{~m}(L) \times 0.005 \mathrm{~m}(H) \times 0.005 \mathrm{~m}(B)$; beam (2) is $0.05 \mathrm{~m}(L) \times 0.005 \mathrm{~m}(H) \times 0.005 \mathrm{~m}(B)$.

beam can be related to its average transverse deflection $\hat{W}$. However, unlike for rigid freestanding beams where the maximum transmitted impulse scales directly to its mass per unit area (or $\beta_{w}$ ) through Eq. 41, the entire temporal-history of the beam transverse velocity must first be determined - this is needed to calculate $\hat{W}$ in Eq. 40 - before the maximum 


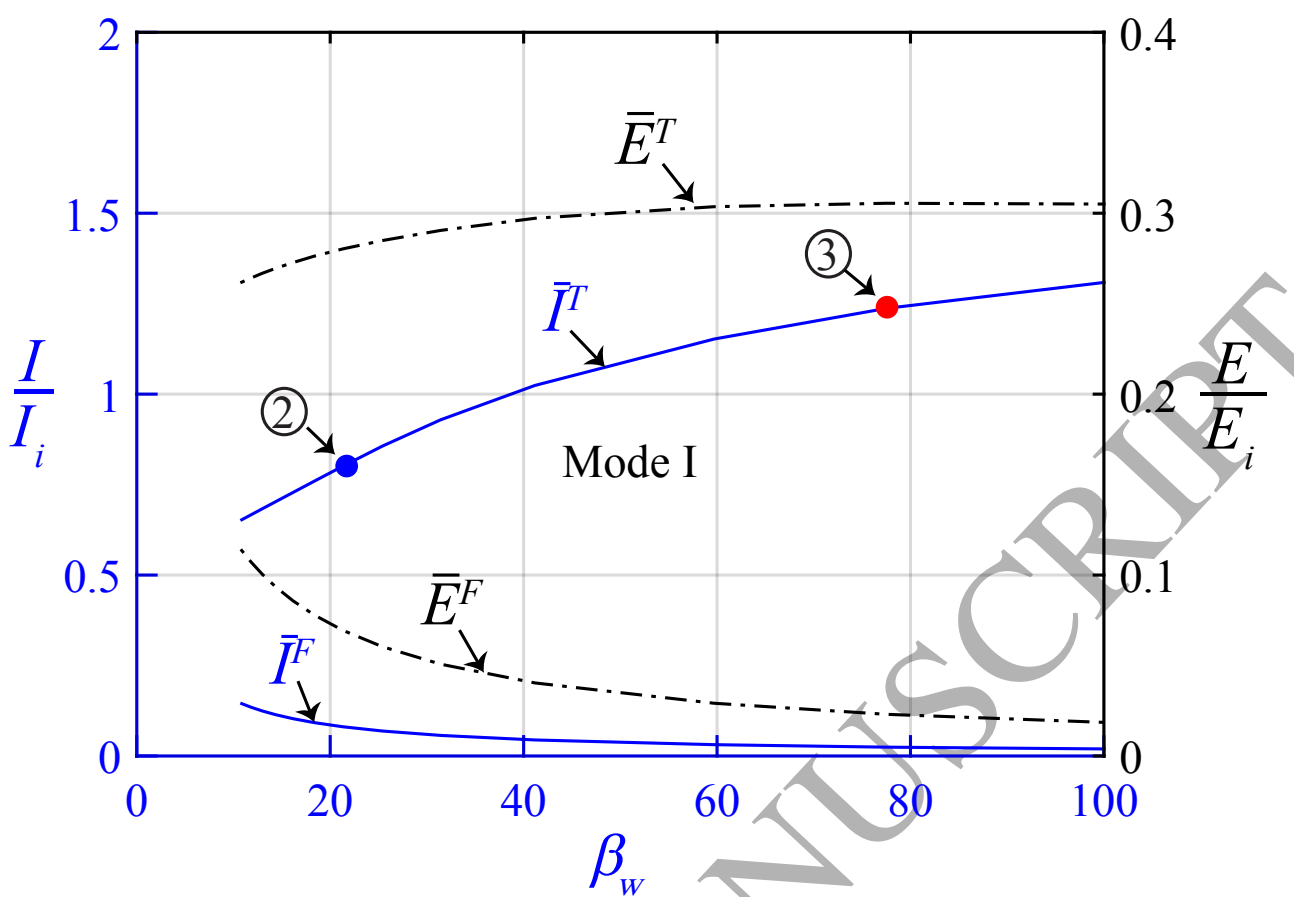

(a)

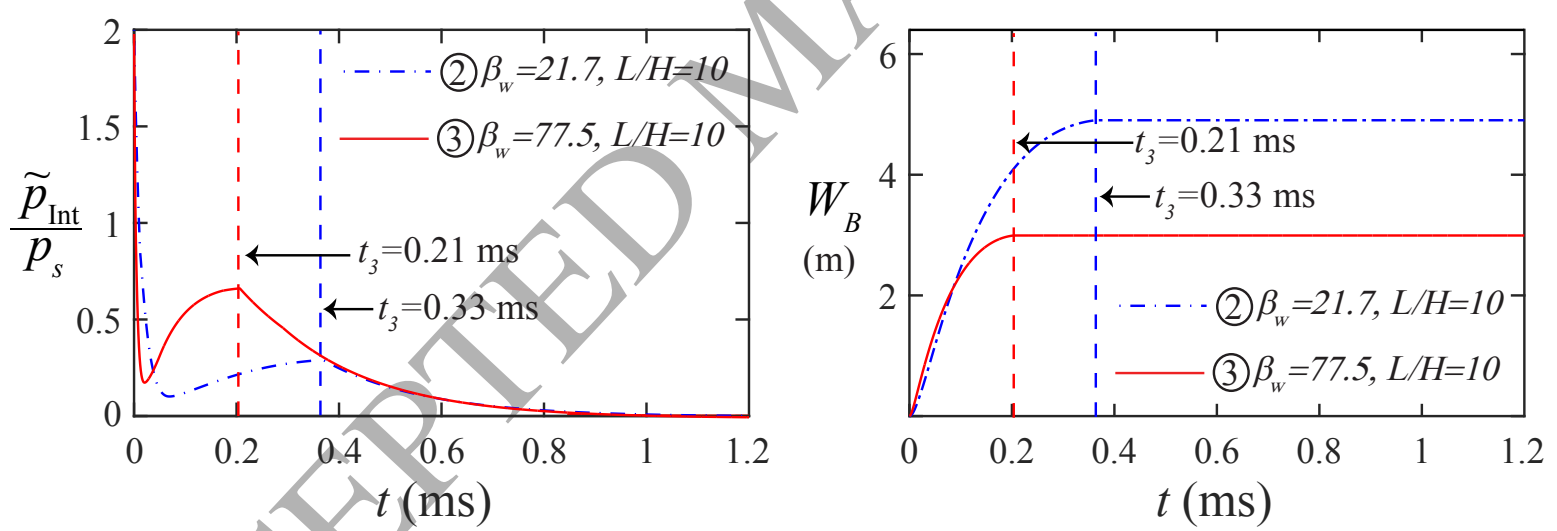

(b)

Figure 12: (a) Analytical prediction of maximum impulse (- corresponds to $\bar{I}^{T}$ and $\bar{I}^{F}$ ) and energy (-.corresponds to $\bar{E}^{T}$ and $\bar{E}^{F}$ ) for elasto-plastic beams with identical aspect ratio $L / H=10$ deforming in mode I; and (b) Comparison of analytical normalised temporal average interface pressure $\tilde{p}_{\text {Int }}(t) / p_{s}$ and temporal mid-span deflection $W_{B}(t)$ for beams (2) and (3) in Fig 12a. Beam (2) is $0.05 \mathrm{~m}(L) \times 0.005 \mathrm{~m}(H)$ $\times 0.005 \mathrm{~m}(B)\left(\beta_{w}=21.7\right)$; beam (3) is $0.014 \mathrm{~m}(L) \times 0.0014 \mathrm{~m}(H) \times 0.0014 \mathrm{~m}(B)\left(\beta_{w}=77.5\right)$.

transmitted impulse can be evaluated.

It is also worth noting that in both Figs 11a and 12a, the elasto-plastic beams have significantly higher non-dimensional maximum transmitted impulse and energy compared to 
free-standing beams. This is because, as alluded to earlier, the fluid-structure interaction time for elasto-plastic beams are significantly longer.

\subsection{Mode II}

Variations of the maximum non-dimensional impulse and energy terms against $L / H$ and $\beta_{w}$ for a peak incident overpressure of $p_{s}=120 \mathrm{MPa}$ are shown in Figs 13 and 14 respectively. Note that all the elasto-plastic beams shown deform in mode II. Figure 13a plots the maximum non-dimensional impulse and energy terms, against the aspect ratio $L / H$ with $\beta_{w}=54.3$. Both $\bar{I}^{T}$ and $\bar{E}^{T}$ do not reduce monotonically with $L / H$. This is because as $L / H$ increases - comparing (1) to (2) in Fig 13b - (1) has a lower average interface pressure $\tilde{p}_{\text {Int }}(t) / p_{s}$ and, consequently, takes longer to reach its maximum mid-span deflection and for complete detachment to occur. Figure 14a shows the effect of mass per unit area, or $\beta_{w}$, on the non-dimensional impulse and energy terms for a fixed aspect ratio $L / H=6$. It shows that both $\bar{I}^{T}$ and $\bar{E}^{T}$ reduce monotonically with $\beta_{w}$ (or less mass per unit area). As opposed to Fig 13 increasing $\beta_{w}$ - compare (2) and (3) in Fig 13 - leads to a reduction in the average interface pressure $\tilde{p}_{\text {Int }}(t) / p_{s}$ and a shorter time is required to reach maximum mid-span beam deflection and for complete detachment to occur.

Increasing $L / H$, as shown in Fig 13b, would lead to a lower $\bar{I}_{2}^{T}$ and a higher $\bar{I}_{3}^{T}$, while increasing $\beta_{w}$ (see Fig 14b) leads to a higher $\bar{I}_{2}^{T}$ and a lower $\bar{I}_{3}^{T}$. This explains the nonmonotonic decay in $\bar{I}^{T}$ in Fig 13a. The monotonic decay in $\bar{I}^{T}$ with $\beta_{w}$ in Fig 14a suggests that the effects of $t_{3}$ on the impulse transmitted in mode II could be more dominant than the transverse beam deflection.

The beneficial effect of FSI for the rigid un-supported beam is evident in Fig 14a. However, this is due to early cavitation triggered by a reduction in the beam's mass per unit area as $\beta_{w}$ increases. Furthermore, the calculation of $\bar{I}^{F}$ has a direct correspondence to the FSI index $\beta_{w}$ given by Eq. 41, as opposed to its deformable and supported counterparts that deform in mode II.

The maximum non-dimensional transmitted impulse and energy are significantly smaller in mode II compared to mode I for the fully-clamped elasto-plastic beams due to a loss of integrity at the supports. Notwithstanding, the maximum non-dimensional transmitted impulse and energy for elasto-plastic beams are still considerably greater than those of freestanding beams since $\bar{I}^{T}>\bar{I}^{F}$ and $\bar{E}^{T}>\bar{E}^{F}$, as is evident in Figs 13a and 14a.

\subsection{Mode III}

Figure 15 plots the non-dimensional maximum impulse and energy for beams deforming in mode III $\left(p_{s}=160 \mathrm{MPa}\right)$. Note that the ratio of the plastic work absorbed through shearing deformation to the total plastic work done has reached the critical ratio of $\beta_{c}=0.45$ in all cases. The results in Fig 15 are broadly similar to Figs 13a and 14a, despite a slight decrease in $\bar{I}^{T}$ and $\bar{E}^{T}$ due to the fact that the time it takes for complete detachment to occur is shorter 


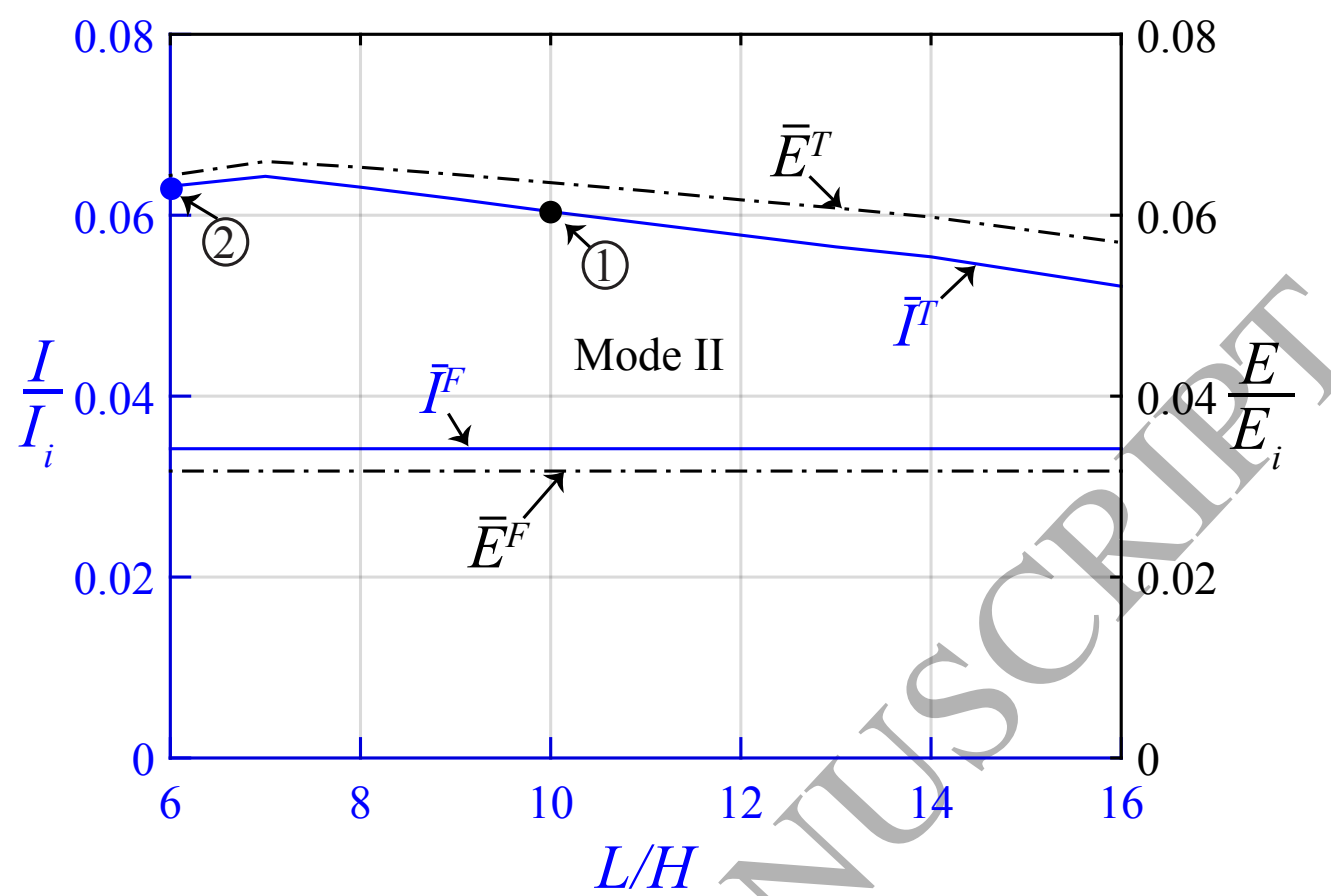

(a)
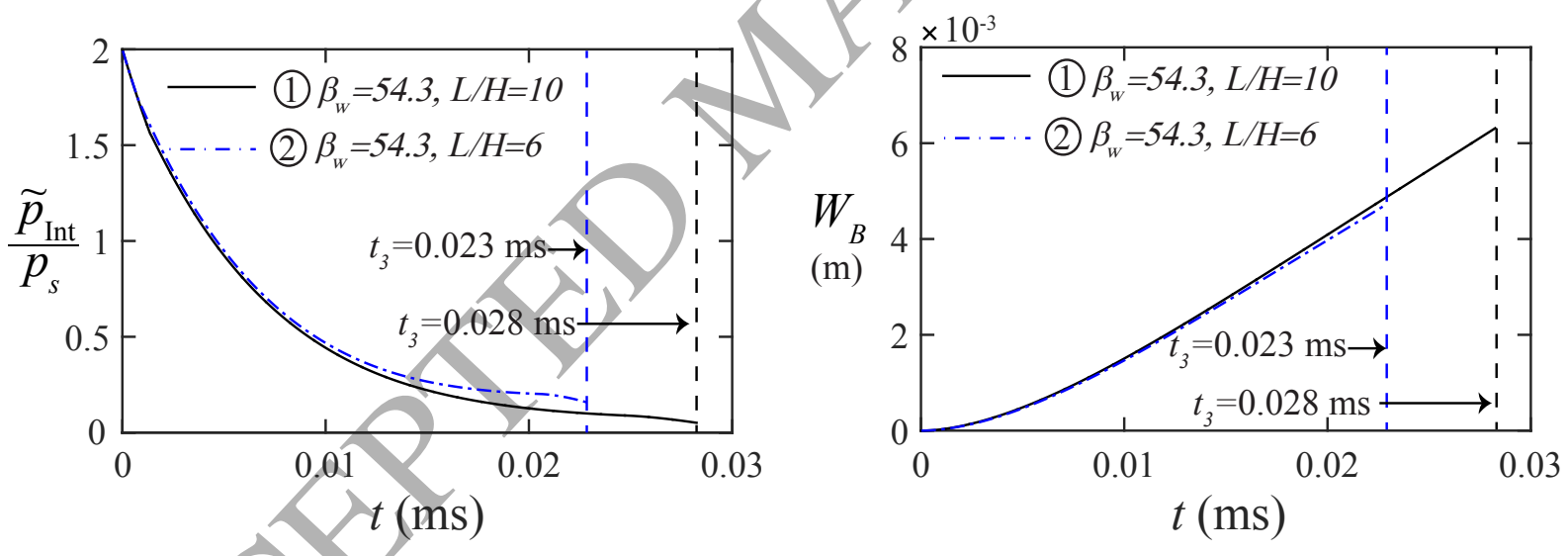

(b)

Figure 13: (a) Analytical prediction of non-dimensional maximum impulse (- corresponds to $\bar{I}^{T}$ and $\bar{I}^{F}$ ) and energy (-.- corresponds to $\bar{E}^{T}$ and $\left.\bar{E}^{F}\right)$ dissipation for elasto-plastic beams with identical cross-section $\left(H=B=0.002 \mathrm{~m}\right.$ ) deforming in mode II where $\beta_{w}=54.3$; and (b) Comparison of analytical normalised temporal average interface pressure $\tilde{p}_{\text {Int }}(t) / p_{s}$ and temporal mid-span deflection $W_{B}(t)$ for beam (1) and (2) in Fig 13a. Beam (1) has $0.02 \mathrm{~m}(L) \times 0.002 \mathrm{~m}(H) \times 0.002 \mathrm{~m}(B)\left(\beta_{w}=54.3\right)$; beam (2) has $0.012 \mathrm{~m}(L)$ $\times 0.002 \mathrm{~m}(H) \times 0.002 \mathrm{~m}(B)\left(\beta_{w}=54.3\right)$.

in mode III than in mode II - compare Figs 14a and 15b for an example. Notwithstanding, the effects of $L / H$ and $\beta_{w}$ on maximum impulse and energy transfer in mode III are broadly the same as that in Figs 13a and 14a for mode II. It is evident from both figures that for 


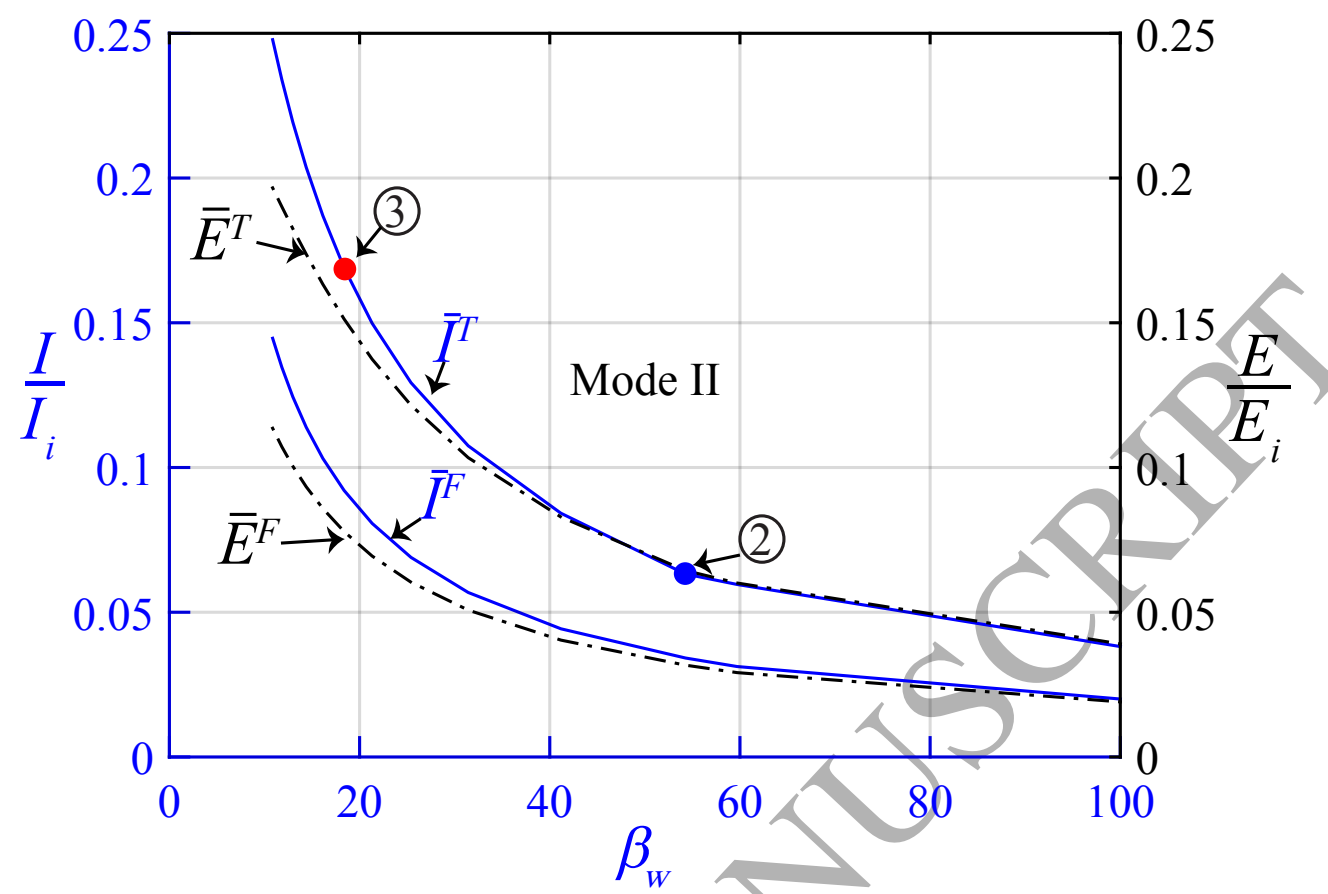

(a)
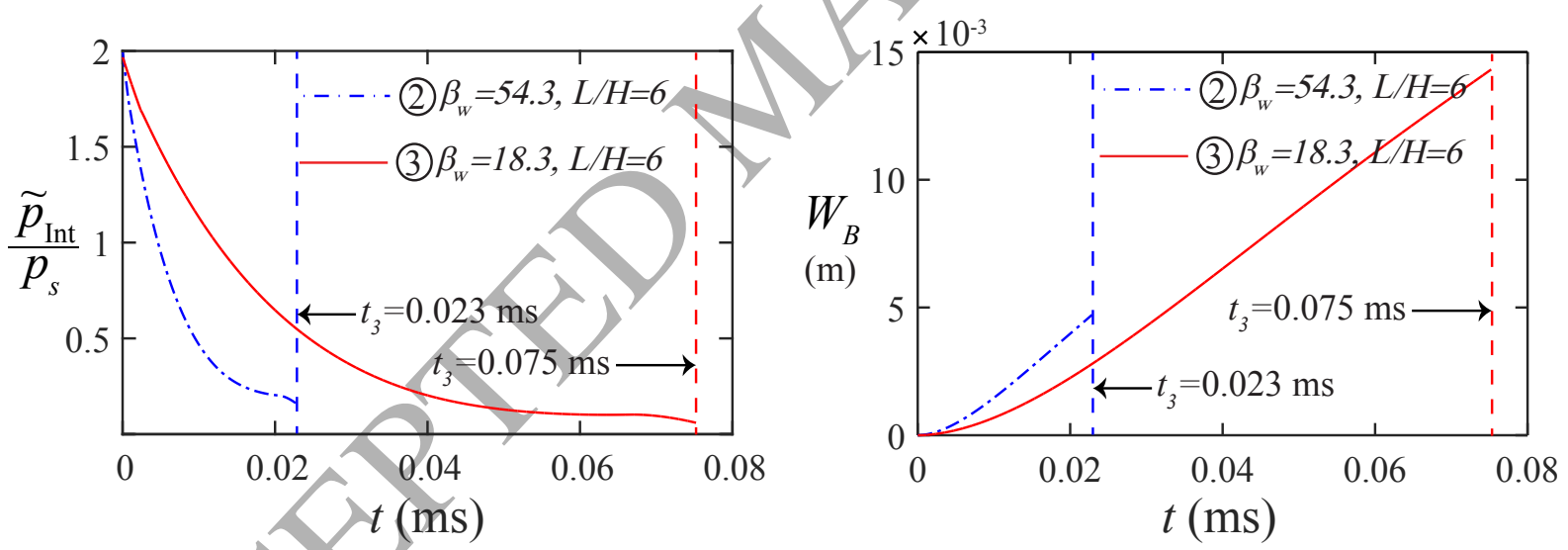

(b)

Figure 14: (a) Analytical prediction of non-dimensional maximum impulse (— corresponds to $\bar{I}^{T}$ and $\bar{I}^{F}$ ) and energy (-.- corresponds to $\bar{E}^{T}$ and $\bar{E}^{F}$ ) dissipation for elasto-plastic beams in mode II with the same aspect ratio $L / H=6$; and (b) Comparison of analytical normalised temporal average interface pressure $\left.\tilde{p}_{\text {Int }}(t)\right\rangle p_{s}$ and temporal mid-span deflection $W_{B}(t)$ for beam (2) and (3) in Fig 13a. Beam (2) is $0.012 \mathrm{~m}(L)$ $\times 0.002 \mathrm{~m}(H) \times 0.002 \mathrm{~m}(B)\left(\beta_{w}=54.3\right)$; and beam (3) is $0.036 \mathrm{~m}(L) \times 0.006 \mathrm{~m}(H) \times 0.006 \mathrm{~m}(B)$ $\left(\beta_{w}=54.3\right)$.

greater impulse and energy were transmitted to the elasto-plastic beams than their freestanding counterparts; this is also the case for modes I and II as shown in Figs 11a, 12a, 13a and 14a. Since both $\bar{I}^{T}$ and $\bar{E}^{T}$ decrease monotonically with peak incident pressure $p_{s}$ in 


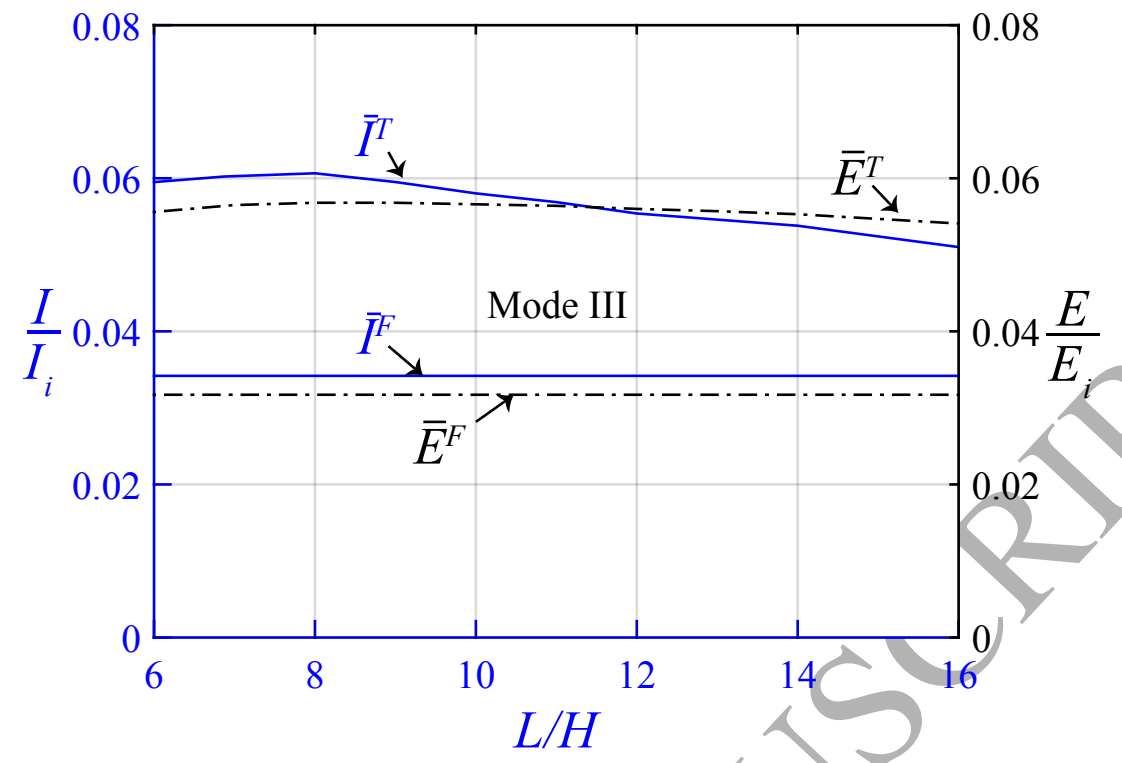

(a)

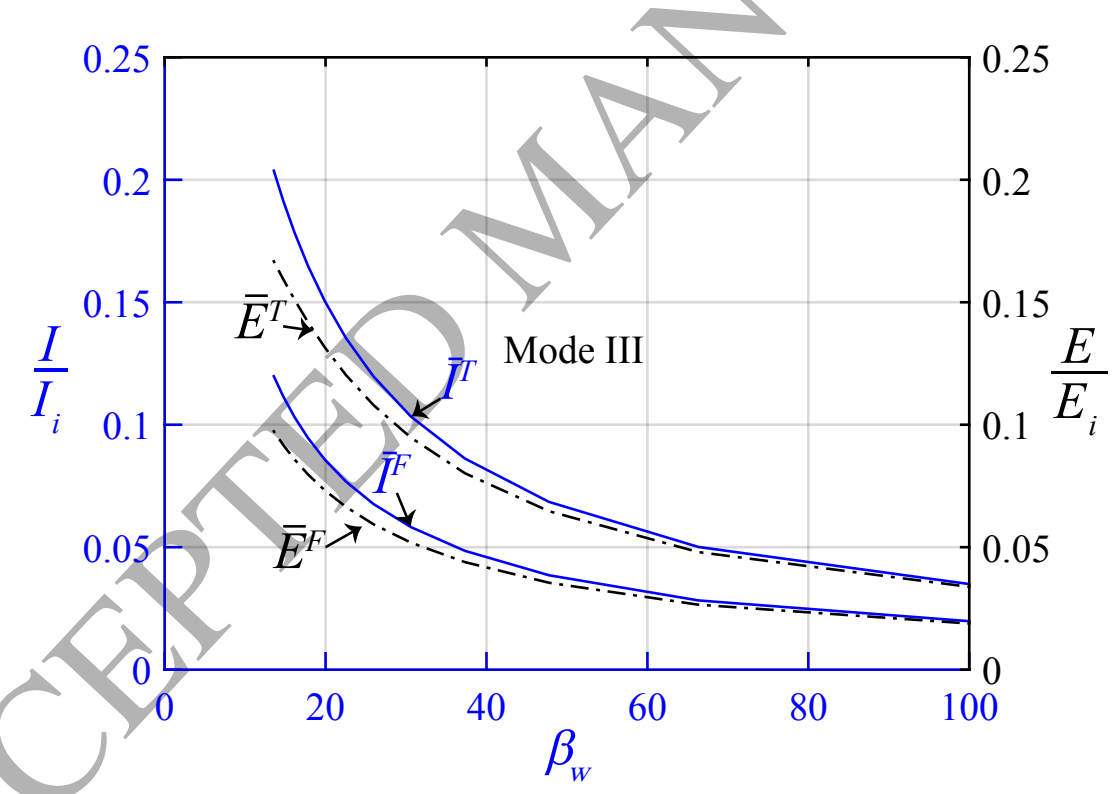

(b)

Figure 15: Analytical predictions of the non-dimensional maximum impulse (— corresponds to $\bar{I}^{T}$ and $\bar{I}^{F}$ ) and energy (-.- corresponds to $\bar{E}^{T}$ and $\bar{E}^{F}$ ) dissipation for two sets of elasto-plastic beams deforming in mode III. (a) of the same cross-section, $H=B=0.002 \mathrm{~m}\left(\beta_{w}=54.3\right)$ and (b) the same aspect ratio, $L / H=6$.

modes II and III (see Fig 10 for an example), maximum transmitted impulse and energy of elasto-plastic beams could be even less than those of rigid, free-standing beams, as a result of significant alleviation of transmitted impulse by boundary failure $\bar{I}_{2}^{T}$. 


\section{Conclusions}

The dynamic response of fully-clamped elasto-plastic beam subjected to underwater blast has been investigated. An analytical model by [1] is used to predict beam deformation, interface pressure history, impulse and energy transfer in a Type I cavitation event where predictions were found to be in excellent agreement with 3D FE simulations. It was found that increasing non-dimensional impulse $I^{*}$ leads to a reduction in the maximum mid-span deflection during modes II and III deformation. A sharp reduction in the maximum transmitted impulse accompanies the transition from mode $\mathrm{I} \rightarrow \mathrm{II}$ and a monotonic reduction in both the maximum transmitted impulse and energy were found in modes II and III. The beneficial effect of FSI in reducing the maximum transmitted impulse to elasto-plastic beams (for a given incident pressure-pulse) was found to be dependent on two parameters: the average beam transverse deflection and the time it takes for complete detachment to occur. The latter significantly limits the impulse imparted to a structure that deforms in modes II and III but not when it is deforming in mode I. The effects of aspect ratio $L / H$ and FSI index $\beta_{w}$ were investigated. It was found that in mode $\mathrm{I}$, an increase in aspect ratio $L / H$, or decrease in FSI index $\beta_{w}$, always leads to a reduction in the maximum impulse transmitted. In modes II and III, increasing FSI index $\beta_{w}$ always leads to a reduction in the maximum transmitted impulse. The effects of boundary on FSI were also investigated where it was shown, as to be expected, that a significantly higher impulse and energy are transmitted to the fully clamped deformable beams, deforming in all three modes, as opposed to their free-standing counterparts of the same mass per unit area.

\section{Acknowledgment}

The authors are grateful to DE\&S Sea Systems Group (Mr. David Manley - Ministry of Defence, UK) and Lloyd's Register Marine (Dr. Fai Cheng - Head of Strategic Research and Technology Policy) for financial support. This work is also funded, in part, by the EPSRC under grant number EP/T028811/1.

\section{Appendix A - Strain energy components in the equation of motion (Eq. 25)}

The dynamic response of the beam system is divided into three separate phases according to [1] and the strain energy components corresponding to each are derived below. Figure 16 presents the flow chart on the numerical implementation of the analytical model. Note that each pháse of motion has its own unique set of initial conditions and associated displacement (and velocity) field. The transitional conditions between phases follow the proposal by Symonds et al. [35], which is based on the well-known 'minimum $\Delta_{0}$ technique'. This technique is commonly employed to determine the starting amplitude of the 'new' velocity field by minimising the difference in kinetic energies between the velocity fields at the end of the terminating phase and at the start of the new phase [13-15]. 


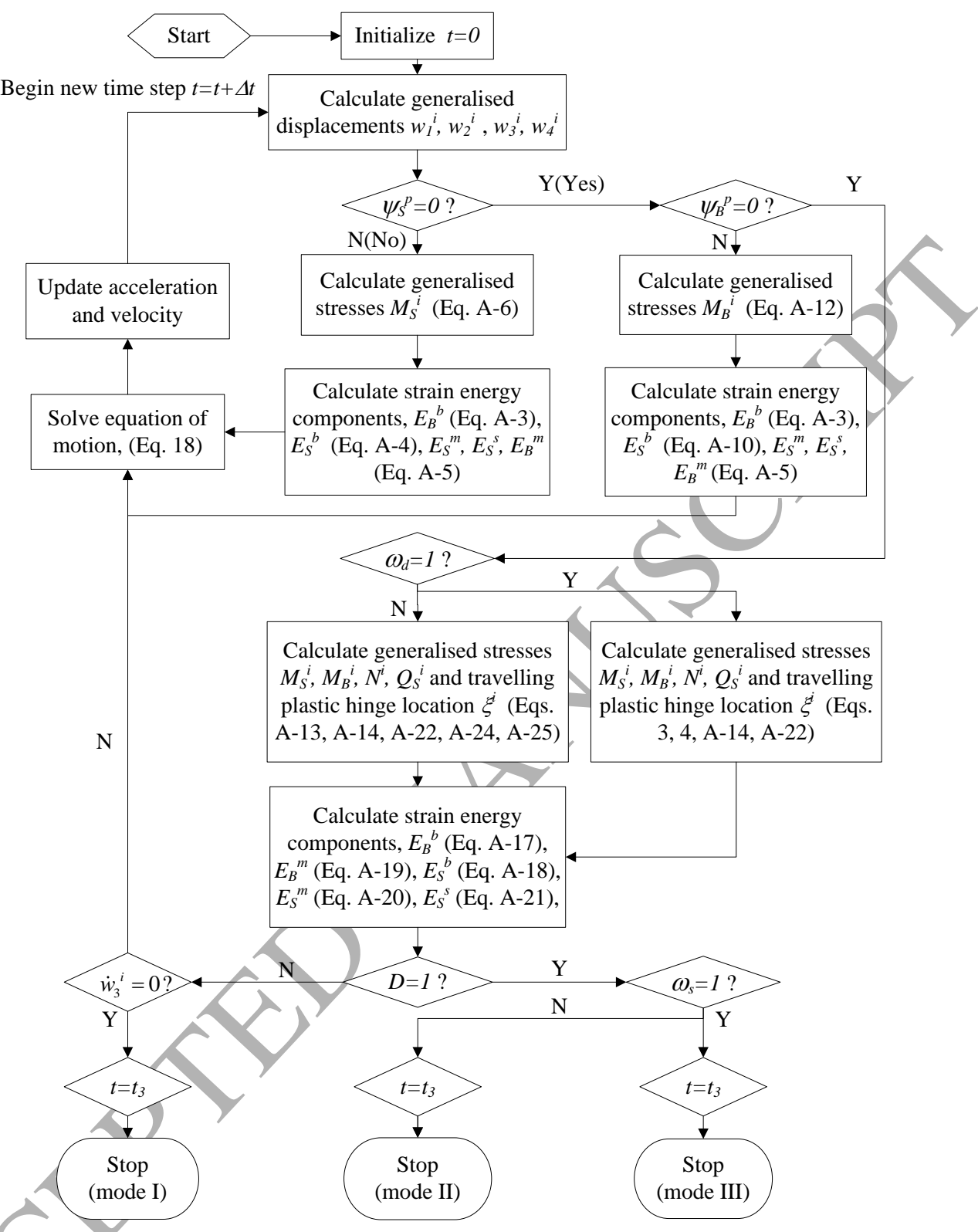

Figure 16: Flow-chart on the numerical implementation of the analytical model in Section 2.

Phase I: $0<t \leq t_{1}$

In Phase I, it is reasonable to neglect catenary actions and transverse shear since the transverse deflection is small compared to the beam thickness, i.e. $W_{B} \ll H[13,36]$. The plastic limit function at the support is simplified according to Eq. 1

$$
\psi_{S}^{p}=\left|\bar{M}_{S}\right|-1 .
$$

Phase I motion ends when $\psi_{S}^{p}=0$. 
and

$$
E_{B}^{b}(t)=\frac{E I}{2} \int_{0}^{L}\left[\frac{\partial^{2} W(x, t)}{\partial x^{2}}\right]^{2} \mathrm{~d} x
$$

$$
E_{S}^{b}(t)=\frac{K_{\phi}}{2} \phi(t)^{2}
$$

where $\phi(t)=\int_{0}^{L}\left[\partial^{2} W(x, t) / \partial x^{2}\right] \mathrm{d} x[13,14]$. Since $W_{B} \ll H$, it is reasonable to assume that the strain energies due to shear and membrane are negligibly small [36]; hence,

$$
E_{S}^{s}(t)=E_{S}^{m}(t)=E_{B}^{m}(t) \simeq 0
$$

Substituting Eqs. A-3, A-4 and A-5 into Eq. 25, and using the initial conditions $w_{1}=w_{2}=0$ and $\dot{w}_{1}=\dot{w}_{2}=0$ gives the two equations of motion for this phase. When the fully plastic stress condition at the support is reached, i.e.

$$
\psi_{S}^{p}=\left|K_{\phi} \phi(t)\right| \mid M_{\varnothing}-1=0,
$$

it marks the end of Phase I deformation at the corresponding time of $t=t_{1}$.

Phase II: $t_{1}<t \leq t_{2}$

In Phase II, small transverse deflection is also assumed [36-38]; hence, the influence of transverse shear and catenary actions are also ignored. Therefore, the fully plastic stress condition at the support- since $\psi_{S}^{p}=0$ - reduces to

$$
\left|\bar{M}_{S}\right|=1
$$

whilst the same at the mid-span simplifies to

$$
\psi_{B}^{p}=\left|\bar{M}_{B}\right|-1 .
$$

\section{Phase It motion ends when $\psi_{B}^{p}=0$.}

An admissible transverse displacement field for Phase II motion, shown in Fig 3b, is given by $[13]$

$$
W(x, t)=\left[w_{1}\left(t_{1}\right)+w_{2}(t)\right] \cos \frac{\pi x}{2 L}
$$

where $w_{1}\left(t_{1}\right)$ is the terminating amplitude of the generalised displacement from Phase I at $t_{1}$. Expressions for strain energy components are identical to those previously derived for 
Phase I (Eq. A-3 for $E_{B}^{b}$; Eq. A-5 for $E_{B}^{m}, E_{S}^{s}$ and $E_{S}^{m}$ ) with the notable exception of the bending strain energy of the rotational spring at the support which is as follows:

$$
E_{S}^{b}(t)=\left|M_{S}\right|\left[\phi(t)-\phi_{1}\right]=M_{0}\left[\phi(t)-\phi_{1}\right]
$$

where $\phi_{1}=M_{0} / K_{\phi}$. Substituting Eqs. A-3, A-5 and A-10 into Eq. 25 gives the equation of motion for Phase II. Following [35], the starting amplitude of the generalised velocity in this phase is given by

$$
\dot{w}_{2}=\frac{8}{3 \pi} \dot{w}_{1}\left(t_{1}\right)+\dot{w}_{2}\left(t_{1}\right)
$$

where $\dot{w}_{1}\left(t_{1}\right)$ and $\dot{w}_{2}\left(t_{1}\right)$ refer to the terminating amplitude of the generalised yelocity from Phase I at time $t_{1}$. Once the mid-span of the beam meets the fully plastic stress condition, i.e.

$$
\psi_{B}^{p}=E I \kappa(t) / M_{0}-1=0
$$

where $\kappa(t)=\partial^{2} W(x, t) /\left.\partial x^{2}\right|_{x=0}$ is the curvature at the mid-span, it marks the end of Phase II deformation at the corresponding time of $t=t_{2}$.

Phase III: $t_{2}<t \leq t_{3}$

The fully plastic stress conditions at the support and míd-span of the beam are, respectively,

$$
\begin{aligned}
\left|\bar{M}_{S}\right|\left(1-\bar{Q}_{S}^{2}\right)+\bar{N}^{2}+\bar{Q}_{S}^{2}=1, & \text { if } & \left|\bar{M}_{S}\right|>0 \\
\bar{N}^{2}+\bar{Q}_{S}^{2}=1, & \text { if } & \left|\bar{M}_{S}\right|=0
\end{aligned}
$$

and

$$
\bar{M}_{B}+\bar{N}^{2}=1 .
$$

If motion of the beam member ceases when the damage variable $D<1$ (Mode I deformation), this will be followed by residual elastic vibration. By contrast, if this happens when $D=1$ then the beam would fail in either mode II or mode III.

The admissible transverse displacement field at the start of Phase III motion is

$$
W(x, t)=\left\{\begin{aligned}
w_{1}\left(t_{1}\right)+w_{2}\left(t_{2}\right)+w_{3}(t) & \text { if } 0 \leq x \leq L-\xi \\
w_{4}(t)+\left[w_{1}\left(t_{1}\right)+w_{2}\left(t_{2}\right)+w_{3}(t)-w_{4}(t)\right] \frac{L-x}{\xi} & \text { if } L-\xi<x<L
\end{aligned}\right.
$$

where $w_{1}\left(t_{1}\right)$ and $w_{2}\left(t_{2}\right)$ are terminating amplitudes of the generalised displacements from Phases I (at time $t_{1}$ ) and II (at time $t_{2}$ ), respectively. When the travelling plastic hinge reaches the mid-span, i.e. $\xi=L$, the admissible transverse displacement field becomes

$$
W(x, t)=w_{4}(t)+\left[w_{1}\left(t_{1}\right)+w_{2}\left(t_{2}\right)+w_{3}(t)-w_{4}(t)\right] \frac{L-x}{L} .
$$


and

$$
E_{S}^{b}(t)=\left|M_{S}\right| \frac{w_{3}(t)-w_{4}(t)}{\xi} .
$$

The bending strain energies of the beam member and rotational spring are, respectively,

$$
E_{B}^{b}(t)=M_{B} \frac{w_{3}(t)-w_{4}(t)}{\xi},
$$

The membrane strain energies of the beam member and the horizontal axial spring may be expressed, respectively, as

$$
E_{B}^{m}(t)=N \Delta_{B}(t)
$$

and

$$
E_{S}^{m}(t)=N \Delta_{S}(t)
$$

where $\Delta_{B}(t)=\Delta(t) /\left(1+1 / \sqrt{1-Q_{S}^{2} / Q_{0}^{2}}\right)$ is the in-plane membrane displacement at the midspan, $\Delta(t)=\left[w_{3}(t)-w_{4}(t)\right]^{2} / \xi$ is the total membrane displacement and $\Delta_{S}(t)=\Delta(t)-\Delta_{B}(t)$ is the membrane displacement at the support. Here, it is assumed that in-plane membrane displacement is significant where a plastic hinge has developed. The shear strain energy of the vertical axial spring is

$$
E_{S}^{s}(t)=Q_{S} w_{4}(t)
$$

Note that the parameters $M_{S}, Q_{S}, N, M_{B}$, $\xi$ and $\xi$ in Eqs. A-17, A-19, A-18, A-20 and A-21 are unknowns. Recasting them in a non-dimensional form, viz. $\bar{M}_{S}, \bar{Q}_{S}, \bar{N}, \bar{M}_{B}, \bar{\xi}=\xi / L$ and $\dot{\bar{\xi}}=\dot{\xi} / L$, they will have to be computed as described below.

The non-dimensional velocity of the travelling hinge $A$ is [20]

$$
\dot{\bar{\xi}}=\frac{\sigma_{Y}}{\rho L^{2}} \frac{1.5\left(\left|\bar{M}_{S}\right|+\bar{M}_{B}\right)-2 \bar{Q}_{S} \dot{\bar{\xi}}(L / H) / \sqrt{3}+6 \bar{N}\left(\bar{w}_{3}-\bar{w}_{4}\right)+\bar{\xi}\left[p(t) / p_{c}\right]}{\bar{\xi}\left(\dot{\bar{w}}_{3}-\dot{\bar{w}}_{4}\right)}
$$

where $\bar{w}_{3}=w_{3}(t) / H, \dot{\bar{w}}_{3}=\dot{w}_{3}(t) / H, \dot{\bar{w}}_{4}=\dot{w}_{4}(t) / H ; p_{c}=4 M_{0} / L^{2}$ is the fully plastic collapse force per unit length, i.e. the largest force per unit length that can be supported by the structural beam system when subjected to a pure bending moment before the bending moment at each plastic hinge reaches the fully plastic bending moment $M_{0}$ [20]. Note that when the travelling hinge $A$ reaches the existing stationary hinge at the mid-span $B$, they coalesce into a/single stationary hinge so that in subsequent motion

$$
\dot{\bar{\xi}}=0 \quad \text { and } \quad \bar{\xi}=1 \text {. }
$$

The calculations of $\bar{M}_{S}, \bar{Q}_{S}, \bar{N}, \bar{M}_{B}$ would depend on whether damage had initiated. If the state variable $\omega_{d}<1$, then $\bar{M}_{S}, \bar{Q}_{S}$ and $\bar{N}$ are governed by normality requirements so that plastic flow must occur at a non-negative energy dissipation rate since they have met the 
fully plastic stress condition (Eq. A-13). Therefore, according to [20],

$$
\begin{array}{r}
\bar{N}\left(1+\frac{1}{\sqrt{1-\bar{Q}_{S}^{2}}}\right)=2\left(\bar{w}_{3}-\bar{w}_{4}\right) \\
\bar{Q}_{S}\left[\frac{2}{\sqrt{1-\bar{Q}_{S}^{2}}}-\frac{\left|\bar{M}_{S}\right|}{\sqrt{1-\bar{Q}_{S}^{2}}}\right]=\frac{4 \bar{\xi}(L / H) \dot{\bar{w}}_{4}}{\sqrt{3}\left(\dot{\bar{w}}_{3}-\dot{\bar{w}}_{4}\right)}
\end{array}
$$

if $\left|\bar{M}_{S}\right|>0$, and

$$
\bar{N}\left[\frac{2 \dot{\bar{w}}_{4}}{\sqrt{3} \bar{Q}_{S}}+\frac{\dot{\bar{w}}_{3}-\dot{\bar{w}}_{4}}{\bar{\xi}(L / H)}\right]=2 \frac{\left(\bar{w}_{3}-\bar{w}_{4}\right)\left(\dot{\bar{w}}_{3}-\dot{\bar{w}}_{4}\right)}{\bar{\xi}(L / H)}
$$

if $\left|\bar{M}_{S}\right|=0$. If the state variable $\omega_{d}=1$, then the non-dimensional bending moment $\bar{M}_{S}$, membrane force $\bar{N}$ and shear force $\bar{Q}_{S}$ are governed by Eq. 3. Note that the non-dimensional bending moment $\bar{M}_{B}$ remains governed by fully plastic stress condition established for the mid-span $\left(\psi_{B}^{p}=0\right)$ in Eq. A-14.

To calculate $\bar{M}_{S}, \bar{Q}_{S}, \bar{N}$ and $\bar{M}_{B}$, they have to be expressed as functions of $\bar{w}_{3}, \bar{w}_{4}, \dot{\bar{w}}_{3}, \dot{\bar{w}}_{4}$, $\bar{\xi}, \dot{\bar{\xi}}$ and $t$ through Eqs. A-13, A-14, A-22, A-24 and A-25 if $\omega_{d}<1$; and through Eqs. 4, $3, \mathrm{~A}-14$ and A-22 if $\omega_{d}=1$. The temporal evolution of these parameters are obtained by solving the aforementioned equations using the well-known 4th order Runge-Kutta method with the initial conditions of $w_{3}=w_{4}=0$ and $\dot{w}_{4}=0$. The starting amplitude of the generalised velocity $\dot{w}_{3}$ for Phase III is given by

$$
\dot{w}_{3}=\frac{12}{\pi^{2}} \dot{w}_{2}\left(t_{2}\right)
$$

where $\dot{w}_{2}\left(t_{2}\right)$ refers to the terminating amplitude of the generalised velocity from Phase II at time $t_{2}$. Phase III deformation ends at time $t_{3}$ if motion of the beam member ceases i.e.

$$
\dot{w}_{3}\left(t_{3}\right)=0 .
$$

If the damage variable $D<1$ when this occurs, then the beam fails in mode I. Otherwise, a mode II or mode HI failure would ensue if $D=1$.

\section{References}

[1] Y. Yuan, P. Tan, K. Shojaei, P. Wrobel, Large deformation, damage evolution and failure of ductile structures to pulse-pressure loading, Int. J. Solids Struct. 96 (2016) 320 - 339. doi:10.1016/j. ijsolstr.2016.05.019.

URL http://www.sciencedirect.com/science/article/pii/S0020768316301068

[2] G. I. Taylor, The Pressure and Impulse of Submarine Explosion Waves on Plates. In The Scientific Papers of Sir Geoffrey Ingram Taylor, Cambridge: Cambridge University Press, 1941.

[3] G. J. McShane, V. S. Deshpande, N. A. Fleck, The underwater blast resistance of metallic sandwich beams with prismatic lattice cores, J. Appl. Mech. 74 (2) (2006) 352-364. doi:10.1115/1.2198549. URL http://dx.doi.org/10.1115/1.2198549 
[4] G. J. McShane, V. S. Deshpande, N. A. Fleck, Underwater blast response of free-standing sandwich plates with metallic lattice cores, Int. J. Impact Eng. 37 (11) (2010) 1138 - 1149. doi:10.1016/j. ijimpeng. 2010.05.004.

URL http://www.sciencedirect.com/science/article/pii/S0734743X10000941

[5] Z. Xue, J. W. Hutchinson, A comparative study of impulse-resistant metal sandwich plates, Int. J. Impact Eng. 30 (10) (2004) 1283 - 1305. doi:10.1016/j.ijimpeng.2003.08.007. URL http://www.sciencedirect.com/science/article/pii/S0734743X03001039

[6] V. S. Deshpande, N. A. Fleck, One-dimensional response of sandwich plates to underwater shock loading, J. Mech. Phys. Solids 53 (11) (2005) 2347 -2383. doi:10.1016/j.jmps.2005.06.006. URL http://www.sciencedirect.com/science/article/pii/S0022509605001213

[7] N. A. Fleck, V. S. Deshpande, The resistance of clamped sandwich beams to shock loading, J. Appl. Mech. 71 (3) (2004) 386-401. doi:10.1115/1.1629109. URL $10.1115 / 1.1629109$

[8] M. T. Tilbrook, V. S. Deshpande, N. A. Fleck, Underwater blast loading of sandwich beams: Regimes of behaviour, Int. J. Solids Struct. 46 (17) (2009) 3209 - 3221. doi:10.1016/j.ijsolstr.2009.04.012. URL http://www.sciencedirect.com/science/article/pii/S0020768309001747

[9] L. F. Mori, D. T. Queheillalt, H. N. G. Wadley, H. D. Espinosa, Deformation and failure modes of i-core sandwich structures subjected to underwater impulsive loads, Exp. Mech. 49 (2) (2009) 257-275. doi:10.1007/s11340-008-9166-9.

URL http://dx.doi.org/10.1007/s11340-008-9166-9

[10] E. H. Kennard, Cavitation in an elastic liquid, Phys. Rev. 63 (1943) 172-181. doi:10.1103/PhysRev. 63.172 . URL http://link.aps.org/doi/10.1103/PhysRev.63.172

[11] A. Schiffer, V. L. Tagarielli, N. Petrinic, A. C. F. Cocks, The response of rigid plates to deep water blast: Analytical models and finite element predictions, J. Appl. Mech. 79 (6) (2012) 061014-061014. doi:10.1115/1.4006458.

URL http://dx.doi.org/10.1115/1.4006458

[12] A. Schiffer, V. L. Tagarielli, The response of circular composite plates to underwater blast: Experiments and modelling, J. Fluids Struct. 52 (2015) 130 - 144. doi:10.1016/j.jfluidstructs .2014.10.009. URL http://www.sciencedirect.com/science/article/pii/S0889974614002242

[13] G. K. Schleyer, S. S. Hsu, A modelling scheme for predicting the response of elastic-plastic structures to pulse pressure loading, Int. J. Impact Eng. 24 (8) (2000) 759 - 777. doi:10.1016/S0734-743X (00) 00019-1.

URL http://www.sciencedirect.com/science/article/pii/S0734743X00000191

[14] G. Langdon, G. Schleyer, Inelastic deformation and failure of profiled stainless steel blast wall panels. part ii: analytical modelling considerations, Int. J. Impact Eng. 31 (4) (2005) 371 - 399. doi : 10.1016/ j.ijimpeng. 2003.12.011.

URL http://ww. sciencedirect.com/science/article/pii/S0734743X03002367

[15] W. J. Stronge, T. X. Yu, Dynamic Models for Structural Plasticity, Springer, 1993.

[16] ABAQUS, User's Manual Version 6.10, Dassault Systemes Simulia Corp., Providence, RI. (2010).

[17] H. M. Wen, Deformation and tearing of clamped work-hardening beams subjected to impulsive loading, Int. J. Impact Eng. 18 (4) (1996) 425 - 433. doi : 10.1016/0734-743X (95) 00053-D. URL http://www.sciencedirect.com/science/article/pii/0734743X9500053D

[18] M. Alves, N. Jones, Impact failure of beams using damage mechanics: Part i: analytical model, Int. J. Impact Eng. 27 (8) (2002) 837 - 861. doi:10.1016/S0734-743X (02)00017-9. URL http://www.sciencedirect.com/science/article/pii/S0734743X02000179

[19] S. B. Menkes, H. J. Opat, Broken beams, Exp. Mech. 13 (11) (1973) 480-486. doi:10.1007/ BF02322734.

URL http://dx.doi.org/10.1007/BF02322734

[20] W. Q. Shen, N. Jones, A failure criterion for beams under impulsive loading, Int. J. Impact Eng. 12 (1) (1992) 101 - 121. doi:10.1016/0734-743X (92)90359-2. 
URL http://www.sciencedirect.com/science/article/pii/0734743X92903592

[21] N. Jones, Structural Impact, Cambridge: Cambridge University Press, 1989.

[22] T. X. Yu, F. L. Chen, A further study of plastic shear failure of impulsively loaded clamped beams, Int. J. Impact Eng. 24 (6-7) (2000) 613 - 629. doi :10.1016/S0734-743X (99)00038-X. URL http://www.sciencedirect.com/science/article/pii/S0734743X9900038X

[23] N. Jones, Plastic failure of ductile beams loaded dynamically, J. Eng. Industry 98 (1) (1976) $131-136$. doi:10.1115/1.3438805.

URL http://dx.doi.org/10.1115/1.3438805

[24] T. Nonaka, Some interaction effects in a problem of plastic beam dynamics, J. Appl. Mech. 34 (1967) $623-43$.

[25] G. N. Nurick, G. C. Shave, The deformation and tearing of thin square plates subjected to impulsive loads: an experimental study, Int. J. Impact Eng. 18 (1) (1996) 99 - 116. doi : 10.1016/0734-743X (95) 00018-2.

URL http://www.sciencedirect.com/science/article/pii/0734743X95000182

[26] N. Jones, A theoretical study of the dynamic plastic behavior of beams and plates with finite-deflections, Int. J. Solids Struct. 7 (8) (1971) 1007 - 1029. doi:10.1016/0020-7683(71)90078-3. URL http://www.sciencedirect.com/science/article/pii/0020768371900783

[27] G. S. Langdon, A. Ozinsky, S. C. K. Yuen, The response of partially confined right circular stainless steel cylinders to internal air-blast loading, Int. J. Impact Eng. 73 (2014) 1 - 14. doi:10.1016/j . ijimpeng. 2014.05.002.

URL http://www.sciencedirect.com/science/article/pii/s0734743X14001092

[28] J. H. Williams, Fundamentals of applied dynamic, New York: John Wiley \& Sons, 1996.

[29] J. W. Hancock, A. C. Mackenzie, On the mechanisms of ductile failure in high-strength steels subjected to multi-axial stress-states, J. Mech. Phys. Solids 24 (2) (1976) 147 - 160. doi : $10.1016 / 0022-5096$ (76) 90024-7.

URL http://www.sciencedirect.com/science/article/pii/0022509676900247

[30] G. R. Johnson, W. H. Cook, A constitutive model and data for metals subjected to large strains, high strain rates and high temperatures, in: Proceedings of 7 th International Symposium on Ballistics, Netherlands, pp. 541, 1983.

[31] A. Shojaei, G. Z. Voyiadjis, P. Tan, Viscoplastic constitutive theory for brittle to ductile damage in polycrystalline materials under dynamic loading, Int. J. Plast. 48 (2013) 125 - 151. doi:10.1016/j. ijplas.2013.02.009.

URL http://www.sciencedirect.com/science/article/pii/S0749641913000466

[32] Y. Liang, A. V. Spuskanyuk, S. E. Flores, D. R. Hayhurst, J. W. Hutchinson, R. M. McMeeking, A. G. Evans, The response of metallic sandwich panels to water blast, J. Appl. Mech. 74 (1) (2005) 81-99. doi: $10.1115 / 1.2178837$.

URL http://dx.doi.org/10.1115/1.2178837

[33] Y. Yuan, P. J. Tan, Deformation and failure of rectangular plates subjected to impulsive loadings, Int. J. Impact Eng. 59 (2013) 46 - 59. doi:10.1016/j.ijimpeng.2013.03.009. URL http://www.sciencedirect.com/science/article/pii/S0734743X13000730

[34] M. M. Swisdak, Explosion effects and properties - Part II: explosion effects in water. In Technical report, Naval Surface Weapons Center, DahlgrEn VA, Cambridge: Cambridge University Press, 1978.

[35] P. S. Symonds, H. Kolsky, J. M. Mosquera, Simple elastic-plastic method for pulse loading - comparisons with experiments and finite element solutions, Inst. Phys. Conf. Ser. 70 (1984) 479-486.

[36] B. Izzuddin, A simplified model for axially restrained beams subject to extreme loading, Int. J. Steel Struct. 5 (2005) 421-429.

[37] A. S. Fallah, L. Louca, Pressure-impulse diagrams for elastic-plastic-hardening and softening singledegree-of-freedom models subjected to blast loading, Int. J. Impact Eng. 34 (4) (2007) 823 - 842. doi:10.1016/j.ijimpeng.2006.01.007.

URL http://www.sciencedirect.com/science/article/pii/S0734743X06000212

[38] A. S. Fallah, E. Nwankwo, L. A. Louca, Pressure-impulse diagrams for blast loaded continuous beams 


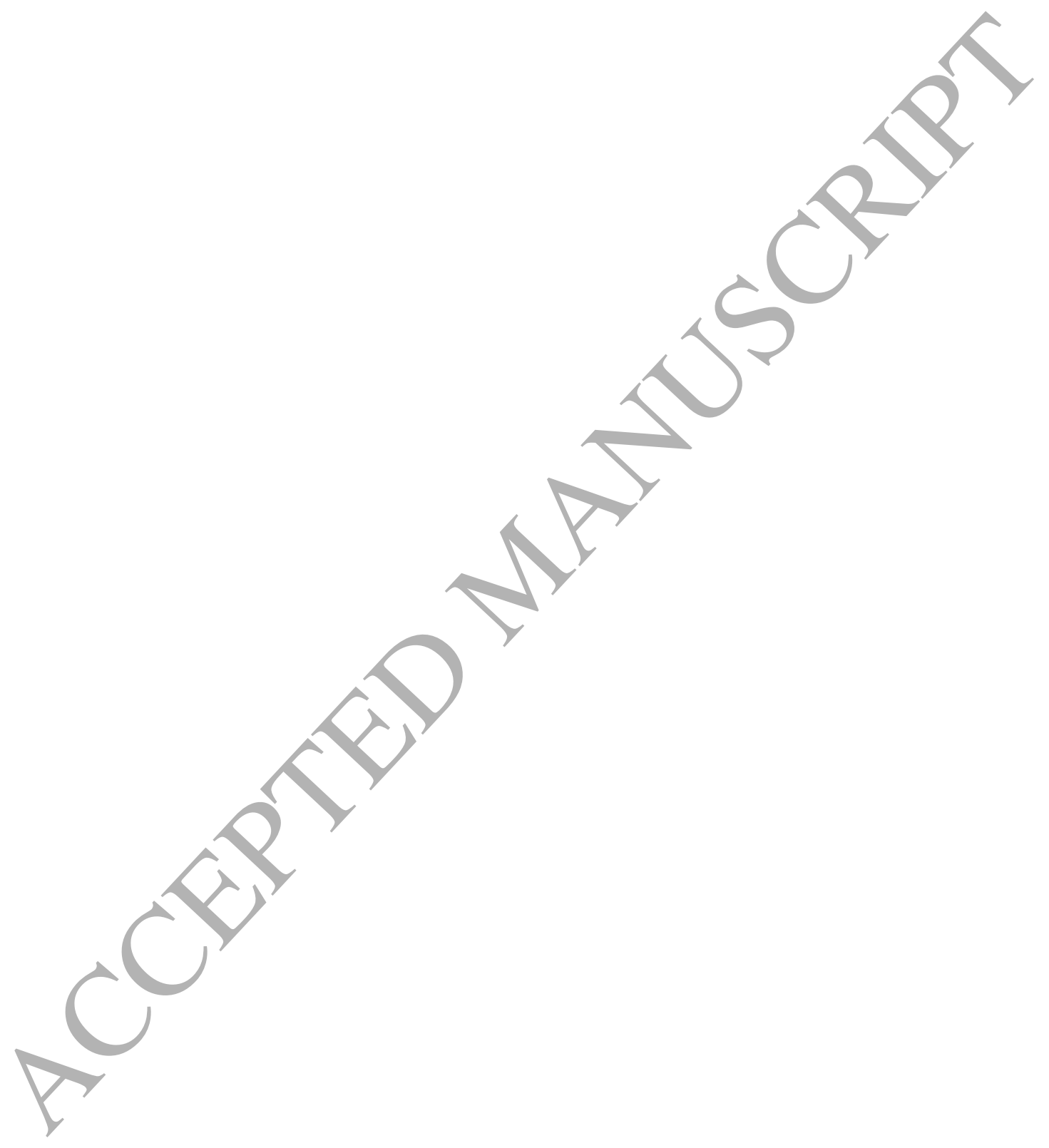

